\title{
Quartäre Vergletscherungen im Hindukusch, Karakorum und West-Himalaya, Pakistan - Ein Überblick
}

\author{
Ulrich Kamp \& Klaus Haserodt*)
}

KAMP, U. \& HASERODT, K. (2002): Quartäre Vergletscherungen im Hindukusch, Karakorum und West-Himalaya, Pakistan - Ein Überblick. - Eiszeitalter und Gegenwart, 51: 93-114; Hannover 2002.

Keywords: Himalaya, Pakistan, Vergletscherung, Pleistozän, Holozän.

Kurzfassung:. Für die pakistanischen Hochgebirge Hindukusch (Chitral, Swat, Ghizar-Gebiet), Karakorum (Hunza, Gilgit-Raum, Rakaposhi, Haramosh, Baltistan, K2-Region) und West-Himalaya (Nanga Parbat, Kaghan-Gebiet, Deosai-Plateau) haben glaziologische und glazialmorphologische Forschungen eine lange Tradition. Mit dem vorliegenden Beitrag wird eine Gesamtschau über den bisherigen Erkenntnisstand zu den quartären Vergletscherungen geliefert. Dabei zeigt sich, dass die Ergebnisse über die Ausdehnung und die glazialen Chronologien nach wie vor differieren - insbesondere bezüglich der Rekonstruktion eines ehemaligen Gletschers im Indus-Tal. Verbesserte Datierungsmethoden revidieren hier ggf. bisherige Ansichten, und neuere Erklärungsmodelle, wie das „Wandern“ des Monsun-Systems während des Quartärs als entscheidender regionaler Klimafaktor, versuchen asynchrone Chronologien zu verstehen.

\section{[Quaternary Glaciations in the \\ Hindu Kush, Karakoram and West-Himalaya, Pakistan - An Overview]}

\begin{abstract}
Glaciological and glaciomorphological studies in the high mountains of Pakistan reflect a long research tradition. A comprehensive review of the current state of research on the Quaternary glaciations in this region is presented: Hindu Kush (Chitral, Swat, Ghizar Region), Karakoram (Hunza, Gilgit Region, Rakaposhi, Haramosh, Baltistan, K2 Region) and WestHimalaya (Nanga Parbat, Kaghan Region, Deosai Plateau). Until today the results about the extent and the glacial chronologies differ, and especially the reconstruction of a former glacier in the Indus valley is still in discussion. Nowadays improved dating methods allow to revise older scientific views, and new models try to explain asynchronous chronologies, like a "shift" of the monsoon during the Quaternary as an important regional climatic factor.
\end{abstract}

\footnotetext{
*)Anschriften der Verfasser: Dr. Ulrich Kamp, Department of Geography and Geology, University of Nebraska at Omaha, 60 ${ }^{\text {th }}$ Dodge Streets, Omaha, NE 68182-0199, Prof. em. Dr. Klaus Haserodt, Gütergotzer Strasse 67, 14165 Berlin.
}

\section{Einleitung}

Die Forschungen zur glazialen Geschichte Hochasiens finden seit langer Zeit Beachtung, und im Lichte der allgemeinen Diskussion um rezente Klimaänderungen (anthropogen beeinflußt oder nicht?) sind sie aktueller denn je. Allerdings gehen die Meinungen über das Ausmaß der quartären Vergletscherungen und die Anzahl der GlazialInterglazial-Zyklen weit auseinander (vgl. BURBANK \& Cheng 1991; Haserodt 1989a u. b; Kuhle 1986, 1991, 1993; SHI YAFENG et al. 1992; ZHENG BeNXING 1989). Die Unsicherheit, die in der Gletscherund damit auch der Klimarekonstruktion liegt, basiert vor allem auf dem Problem einer einwandfreien Identifizierung glazialer Sedimente und ihrer Datierung. Überhaupt sind die glazialen Zeugen innerhalb der Gebirge selbst nur unvollständig erhalten, was insbesondere für Endmoränen der Hochglaziale gilt, und Korrelationen im Rahmen relativer Chronologien bergen immer Gefahren. So können die nur wenigen glazialen Sedimente z. B. irrtümlicherweise einer einzigen Vergletscherung zugeordnet werden, tatsächlich aber verschiedenen Glazialen entstammen. Alle Rekonstruktionen der Vergletscherungsphasen in den Hochgebirgen der Erde insgesamt sind denn auch bisher stets eher grob - angesichts der in Tiefseebohrkernen nachgewiesenen bis zu 32 quartären Vergletscherungen (SHAKLETON \& OPDYKE 1973). Das hier vorgestellte, überarbeitete Schema der jungquartären Chronologien für die nordpakistanischen Hochgebirge nach KAMP (1999) bzw. OwEN (1988) kann demnach nach wie vor nur ein vorläufiges sein, das im Zuge fortschreitender Forschung sicherlich weiterer Ergänzungen und Korrekturen bedarf.

Das Ausmaß der pleistozänen und holozänen Vergletscherungen in Hochasien wird noch immer kontrovers diskutiert. Auf der einen Seite entwarf KuHLE (1989, 1991, 1993) das Modell eines spätpleistozänen Inlandeises über dem tibetischen Plateau nach Art der Verhältnisse im heutigen Grönland und einem randlichen Eisstromnetz, das die umgebenden Gebirge teilweise durchfloss. 


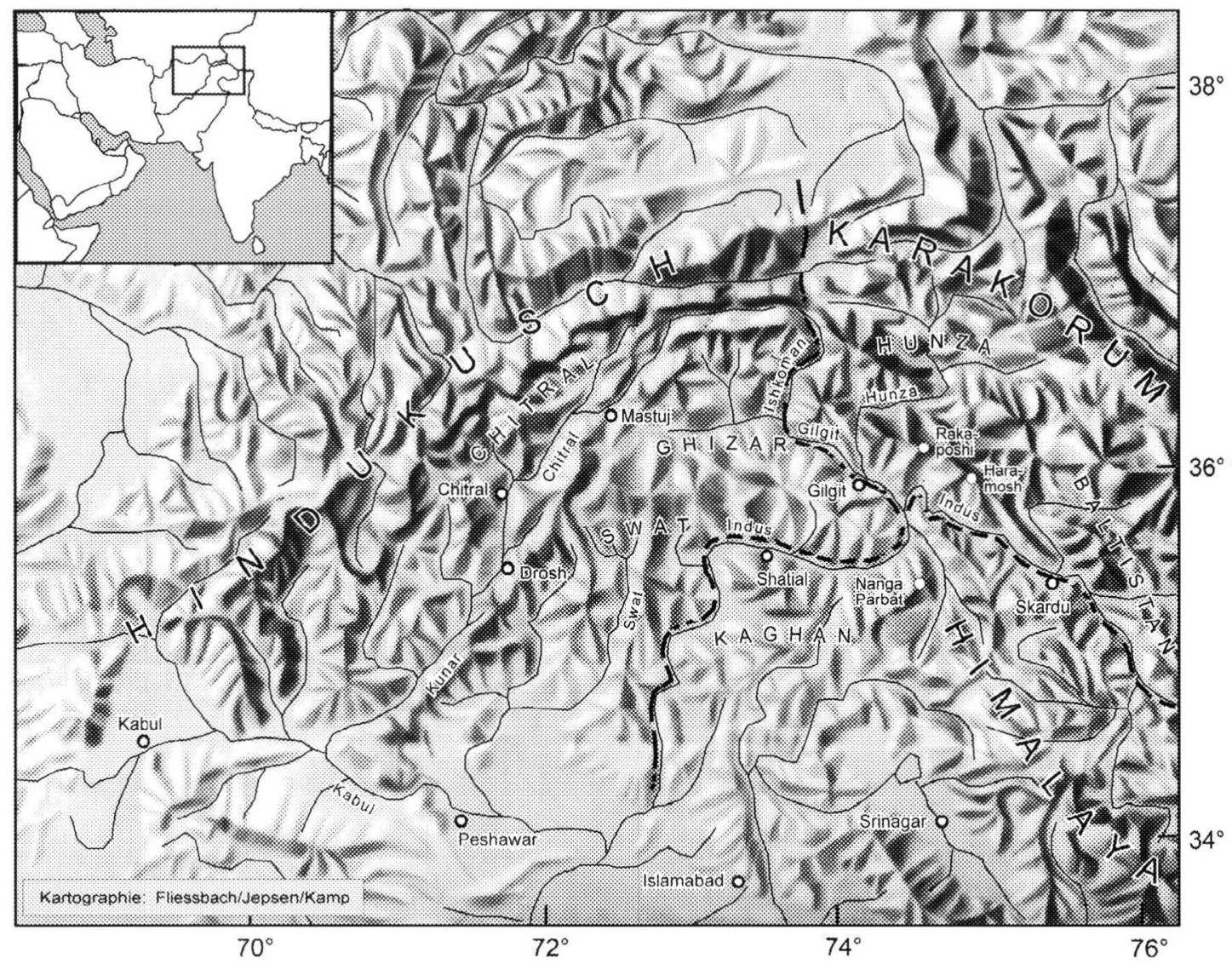

Abb. 1: Die Hochgebirge Nordpakistans: Hindukusch, Karakorum und Himalaya. Die räumliche Abgrenzung der Teilräume (gestrichelte Linie) folgt hier geographischen, nicht geologischen Gesichtspunkten (nach KAMP, 1999, verändert).

Fig. 1: The high mountains of northern Pakistan: Hindu Kush, Karakoram and Himalaya. Here, the spatial demarcation of the subregions (broken line) follows the geographical, not the geological view (after KAMP, 1999, revised).

Auf der anderen Seite vertraten FinSTERWALder (1936) und Troll (1938) sehr früh die Ansicht, dass die Täler des Indus- und des Astor-Flusses im Hochglazial vollständig eisfrei waren. Zwar gilt diese Vorstellung heute als widerlegt, doch zeichnen auch jüngere Forschungsergebnisse das Bild einer - gegenüber dem Modell von KUHLE - eingeschränkteren Vergletscherung. Es scheint sich damit abzuzeichnen, dass das Abbild der pleistozänen Umwelt zwischen den extremen Entwürfen der „Maximalisten“ und der „Minimalisten“ zu suchen ist.
Die räumliche Abgrenzung der in diesem Beitrag beschriebenen Hochgebirge Hindukusch, Karakorum und West-Himalaya ist nicht einfach. So lassen sich sowohl geographische als auch geologische Einheiten (z. B. SEARLE \& KHAN o. J.) herausstellen, die nicht deckungsgleich sind. In dem hier vorliegenden Beitrag sollen die allgemein akzeptierten geographischen Begriffe Verwendung finden. Es werden daher folgende vereinfachende Abgrenzungen entlang von Flüssen vorgenommen (Abb. 1): Die Hindukusch-Kohistan-Region liegt

Abb. 2: Die jungpleistozäne Vergletscherung in Nordpakistan nach HASERoDT (1989b, Neuzeichnung) sowie (*) letztglaziale Maximalausdehnungen nach jüngstem Forschungsstand (nach diversen Autoren, vgl. Text) mit $\left(^{*}\right)$ Datierungslokalitäten $($ Erläuterungen vgl. Tab. 2).

Fig. 2: Extent of Late Pleistocene glaciation in northern Pakistan after HASERODT (1989b, new drawing), as well as $(*)$ late glacial ice maxima (after several authors, cf. text) and (*) dating locations (for explanations cf. Tab. 2). 


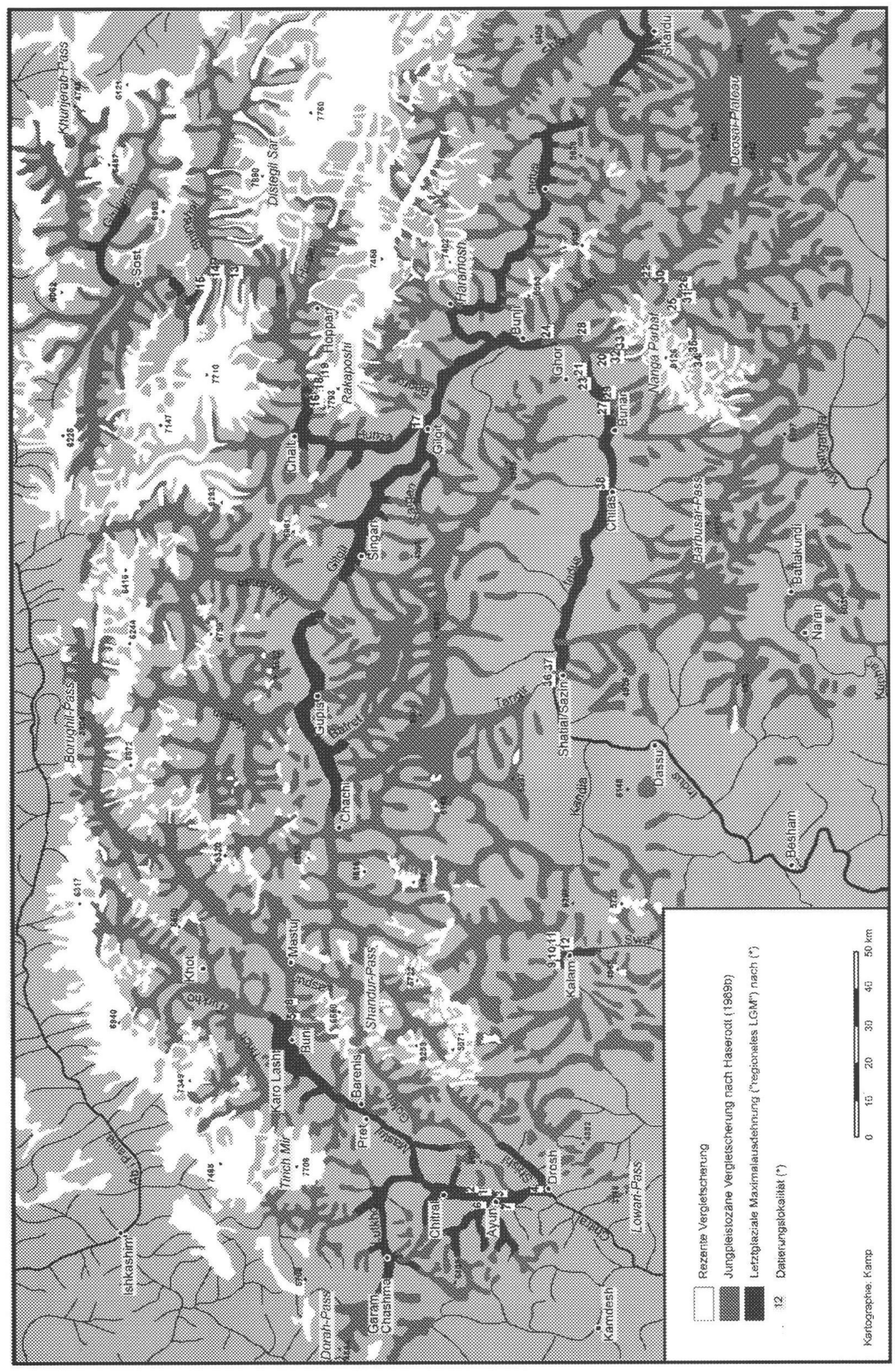


westlich des Ishkoman-, des Gilgit-, des Hunzaund des Indus-Flusses; die Karakorum-Region liegt östlich des Ishkoman-, des Gilgit- und des Hunza- sowie nördlich des Indus-Flusses; der West-Himalaya liegt südlich und östlich des Indus-Flusses. Somit treffen die drei Gebirgsregionen nördlich der Siedlung Bunji (Abb. 2) am Zusammenfluß von Indus und Gilgit aufeinander.

Die mit der Orogenese dieser drei Gebirge verbundenen Hebungsraten, und damit die Frage nach der Beziehung zwischen tektonischer Hebung und pleistozänen Vergletscherungen, stehen noch immer zur Diskussion. Vor allem bereitet hierbei die Berechnung von ehemaligen Hebungsraten aus rezenten Werten Schwierigkeiten. Die gegenwärtigen Hebungsraten wurden für das Kashmir-Bekken mit 4-10 mm/Jahr (BURBANK \& JOHNSON 1982) und für das Zentrum des Nanga Parbat mit 5$10 \mathrm{~mm} / \mathrm{Jahr}$ (ZeItLER et al. 1981, 1982) angegeben. GARDNER \& JONES (1993) bestätigten durch ihre Berechnungen der Denudationsraten des Raikot-Gletschers am Nanga Parbat die von ZeITLeR angegebenen Hebungsraten. BROOKFIELD (1993) kam zu dem Schluß, dass die von anderen Autoren publizierten exponentiell wachsenden $\mathrm{He}$ bungsraten für den Himalaya und angrenzende Gebiete im späten Kanäozoikum zutreffen. Der Beginn der exponentiellen Hebung im frühen Miozän kann für den Himalaya mit seiner Unterschiebung durch die Indische Scholle und mögli- cherweise auch den Karakorum mit einer Unterschiebung durch asiatische Kruste erklärt werden, wobei die Hebungsprozesse allerdings nicht synchron einsetzten: Das Kashmir-Becken in der Region von Srinagar (Abb. 1) z. B. entwickelte sich früher als das Peshawar-Becken, und der in Tadschikistan liegende Pamir hob sich früher als der Karakorum. Zur Veranschaulichung solcher Hebungsaktivität sei hier ein Berechnungsbeispiel für den Hindukusch für den Zeitraum der letzten 100 ky gegeben: Unter der Annahme einer durchschnittlichen Hebungsrate von $5 \mathrm{~mm} / J a h r$ ergäbe sich eine Gebirgshebung von $500 \mathrm{~m}$, und bei Zugrundelegung einer Rate von nur 2,5 mm/Jahr eine Hebung von immerhin noch $250 \mathrm{~m}$.

\section{Vergleichbarkeit von Begriffen und Daten}

Die Durchsicht der vorgestellten Referenzen ergab, dass zum einen nach wie vor bestimmte Begriffe und Definitionen weltweit nicht einheitlich verwendet werden, zum anderen auf die Methodik von Schneegrenzberechnungen oder absoluten Altersdatierungen erst gar nicht eingegangen wird. Auch sind bestimmte Methoden der absoluten Altersdatierung noch immer umstritten. Hinzukommend gehen Datierungslabore u. U. nach unterschiedlichen Analysevorschriften vor, oder neue Erkenntnisse in der Methodik selbst verwerfen ältere Datierungsergebnisse und verlangen nach Neu-Datierungen. All dies erschwert eine ver-

Tab. 1: Relative Chronologien des Quartärs für nordpakistanische Gebirgsräume (aus KAMP, 1999, verändert).

Tab. 1: Relative chronologies of the Quaternary for mountain regions in northern Pakistan (after KAMP, 1999, revised).

\begin{tabular}{|c|c|c|c|c|c|c|c|c|c|c|c|}
\hline \multicolumn{3}{|c|}{ Einteilung } & $\begin{array}{c}\text { Chitral, } \\
\text { Hindukusch }\end{array}$ & \multicolumn{5}{|c|}{$\begin{array}{l}\text { Mittlerer Indus - Gilgit - Hunza, } \\
\text { Karakorum und Himalya }\end{array}$} & $\begin{array}{l}\text { Oberer } \\
\text { Indus }\end{array}$ & \multicolumn{2}{|c|}{$\begin{array}{c}\text { Swat, } \\
\text { Kohistan }\end{array}$} \\
\hline Serie & $\begin{array}{c}\text { Glazial/ } \\
\text { Interglazial }\end{array}$ & Stadial & $\begin{array}{c}\text { KAMP } \\
(1999) / \\
\text { OwEN et al. } \\
(2002)\end{array}$ & $\begin{array}{c}\text { DERBYSHIRE } \\
\text { et al. } \\
(1984)\end{array}$ & $\begin{array}{l}\text { ZHANG } \\
\& \text { SHI } \\
(1980)\end{array}$ & $\begin{array}{c}\text { SHRODER } \\
\text { et al. } \\
(1989)\end{array}$ & $\begin{array}{c}\text { SCHNEIDER } \\
(1959)\end{array}$ & $\begin{array}{l}\text { DESIO \& } \\
\text { OROMBELLI } \\
(1971)\end{array}$ & $\begin{array}{l}\text { CRONIN } \\
(1982)\end{array}$ & $\begin{array}{l}\text { PORTER } \\
(1970)\end{array}$ & \begin{tabular}{|c|} 
RICHARDS \\
et al. \\
$(2000)$
\end{tabular} \\
\hline \multirow{4}{*}{$\begin{array}{l}\text { Holozän } \\
(<13 \mathrm{ka})\end{array}$} & & Rezent & Barum II & Pasu II & Rezent & Rezent & \multirow{4}{*}{$\begin{array}{l}\text { Ältere } \\
\text { Moränen } \\
\text { rezenter } \\
\text { Gletscher }\end{array}$} & & \multirow{7}{*}{$\begin{array}{l}\text { Individuelle } \\
\text { Moränen }\end{array}$} & \multirow{4}{*}{ Neoglazial } & \\
\hline & & Historisch & Barum I & Pasu I & LIA & LIA & & & & & \\
\hline & & \multirow[t]{2}{*}{ Neoglazial } & Shandur II & \multirow[t]{2}{*}{ Batura } & \multirow[t]{2}{*}{ Neoglazial } & \multirow[t]{2}{*}{ Neoglazial } & & & & & \\
\hline & & & Shandur I & & & & & & & & \\
\hline \multirow{9}{*}{$\begin{array}{l}\text { Pleistozän } \\
\text { (13 ka- } \\
2 \text { Mio) }\end{array}$} & \multirow{3}{*}{$\begin{array}{l}\text { Letztes } \\
\text { Glazial }\end{array}$} & & Sonoghar & Gulkhin II & \multirow[b]{3}{*}{ Hunza } & \multirow{2}{*}{$\begin{array}{l}\text { Darel- } \\
\text { Shatial- } \\
\text { Moräne }\end{array}$} & \multirow{2}{*}{$\begin{array}{l}\text { Erdpyrami- } \\
\text { den-Serie }\end{array}$} & \multirow{3}{*}{$\begin{array}{l}\text { Dak-Choki- } \\
\text { Moräne }\end{array}$} & & \multirow[b]{3}{*}{ Kalam I-III } & Ke Gas* \\
\hline & & & Pret & Gulkhin I & & & & & & & Kalam I \\
\hline & & & Drosh & Borit Jheel & & $\begin{array}{l}\text { Dainyor } \\
\text { Moräne }\end{array}$ & $\begin{array}{l}\text { Diluviale } \\
\text { Moränen- } \\
\text { kämme }\end{array}$ & & & & Gabral II \\
\hline & Interglazial & & & \multirow{4}{*}{ Yunz } & \multirow{4}{*}{ Yunz } & Talfüllung III & Alluvium & & \begin{tabular}{|l|} 
Intensive \\
Erosion \\
Alluvium \\
\end{tabular} & \multirow[t]{2}{*}{ Gabral II } & \\
\hline & \multirow{3}{*}{$\begin{array}{l}\text { Mittleres } \\
\text { Glazial }\end{array}$} & Spätes & & & & M2 Tillite & \begin{tabular}{|l} 
Blockschutt- \\
Diamikt \\
Alluvium \\
\end{tabular} & $\begin{array}{l}\text { Gunar- } \\
\text { Moräne }\end{array}$ & Satpura Tillit & & \\
\hline & & & & & & Talfüllung II & \multirow[b]{2}{*}{$\begin{array}{l}\text { Terrassen- } \\
\text { reste }\end{array}$} & & \multirow{4}{*}{$\begin{array}{l}\text { Talfüllung } \\
\text { Bunthang } \\
\text { Tillit }\end{array}$} & & \\
\hline & & Frühes & & & & M1 Tillite & & $\begin{array}{l}\text { Dudishal- } \\
\text { Shatial- } \\
\text { Moränen }\end{array}$ & & Gabral I & \\
\hline & Interglazial & & & \multirow[b]{2}{*}{ Shanoz } & \multirow[b]{2}{*}{ Shanoz } & \begin{tabular}{|l|} 
Obere Jalipur \\
Tal füllung I \\
\end{tabular} & \multirow{2}{*}{ 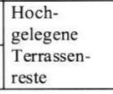 } & & & \multirow[b]{2}{*}{ Laikot } & \\
\hline & $\begin{array}{l}\text { Frühes } \\
\text { Glazial }\end{array}$ & & & & & \begin{tabular}{|l|} 
Unterer \\
Jalipur Tillit \\
\end{tabular} & & & & & \\
\hline
\end{tabular}

* Ke Gas liegt nicht in Swat-Kohistan, sondern im mittleren Indus-Tal. Die Autoren reihen diese Lokalität in ihre Chronologie, die eigentlich nicht nur für Swat-Kohistan, sondern den Himalaya Nordpakistans gelten soll, ein. 


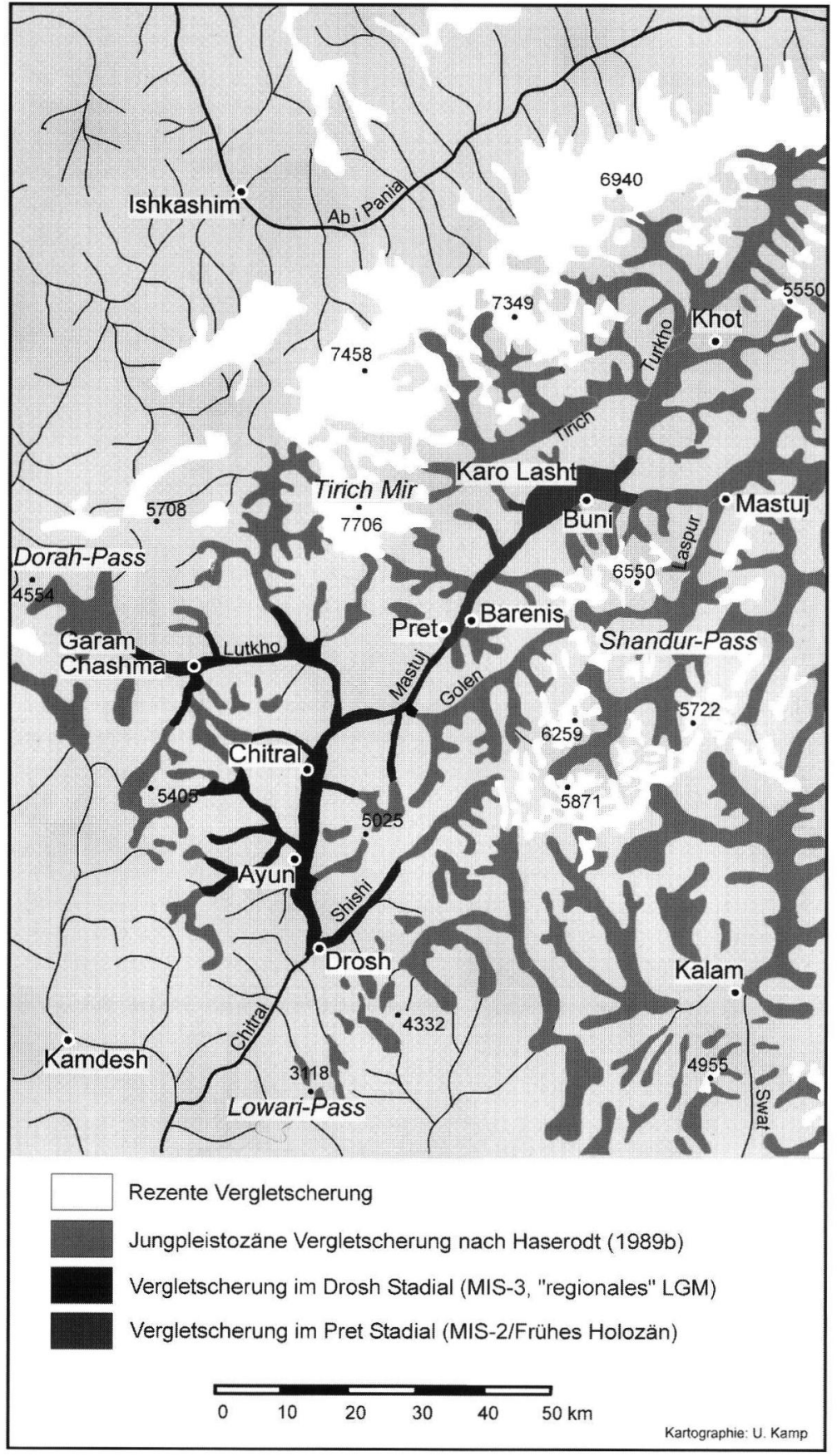

Abb. 3: Eisausdehnung in Chitral, Hindukusch, während des Drosh-Stadials (MIS-3) und Pret-Stadials (MIS-2/Frühes Holozän) nach KAMP (1999, Neuzeichnung) (Alterseinordnung korrigiert nach OwEN et al., 2002).

Fig. 3: Ice extent in Chitral, Hindu Kush, during the Drosh Glacial Stage (MIS-3) and Pret Glacial Stage (MIS-2/Early Holocene) after KAMP (1999, new drawing) (ages corrected by Owen et al., 2002). gleichende Zusammenschau der bisherigen Forschungsergebnisse. Die Anwendbarkeit und Probleme diverser Methodiken können hier jedoch nicht diskutiert werden. In Bezug auf Lumineszenzdatierungen wird auf die Arbeiten von JANOTTA (1991) sowie JANOTTA \& BRUNOTTE (1995) verwiesen, und Berechnungen der Schneegrenze bzw. Equilibrium-Line Altitude (ELA) sind ausführlich in BenN \& LehmKuHL (2000) diskutiert.

Es sei darauf hingeweisen, dass in diesem Überblick über den Forschungsstand lediglich die in der Primärliteratur verwendeten Begriffe und vorgelegten Werte zitiert werden können. Um Verwirrung bei der Verwendung von Begriffen möglichst zu vermeiden, seien zumindest für diesen Artikel folgende Erläuterungen gegeben: Schneegrenze und EquilibriumLine Altitude (ELA): Viele Referenzen nennen die Berechnungsmethodik nicht; in den meisten Fällen mag es sich jedoch um eher gröbere Gletscher-Schneegrenz-Abschätzungen im Sinne von z. B. Heuberger (1980) als um genauere ELA-Kalkulationen nach z. B. Kerschner (1990) handeln.

L ast Glacial Maximum (LGM): Der Begriff wird in der Literatur unterschiedlich verwendet; hier erfährt er daher eine Präzisierung: als ,globales LGM“ (gLGM) wird die Zeitmarke von ca. $20 \mathrm{ky} \mathrm{BP}$ verstanden, als „,regionales LGM“ (rLGM) die jeweilige Maximalausdehnung in einer bestimmten Region; nur bei Unklarheit in der Primärliteratur wird auch hier allgemein von „LGM“ gesprochen. Dies bedeutet für das Spätglazial: Im Falle des gLGM 
Tab. 2: Absolute Altersdatierungen aus den nordpakistanischen Hochgebirgen.

Tab. 2: Results of absolute datings from the mountains of northern Pakistan.

\begin{tabular}{|c|c|c|c|c|c|c|c|c|c|}
\hline $\begin{array}{l}\text { Nr. in } \\
\text { Abb. } 2\end{array}$ & Lokalität & $\begin{array}{l}\text { Geomorphologie } \\
\text { Probenlokalität }\end{array}$ & $\begin{array}{c}\text { Höhe } \\
\text { (m über } \\
\text { Fluß) }\end{array}$ & $\begin{array}{c}\text { Sediment / } \\
\text { Organisches } \\
\text { Material }\end{array}$ & Methode & Alter $(\mathbf{k a})^{+}$ & $\begin{array}{c}\text { Relative } \\
\text { Einordnung }\end{array}$ & $\begin{array}{c}\text { Regionale } \\
\text { Bezeichnung }\end{array}$ & Referenz \\
\hline & Chitral, Hindukusch & & & & & & & & \\
\hline 1 & $\begin{array}{l}\text { Sin Kulum / Broz } \\
(1480 \mathrm{~m})\end{array}$ & $\begin{array}{l}\text { Alluvialer Fächer } \\
\text { unter Schutthang }\end{array}$ & 125 & Fluvial & OSL & $53 \pm 6$ & Letztes Glazial & Broz Periode & OWEN et al. (2002)* \\
\hline 2 & $\begin{array}{l}\text { Zhuti Lasht / Tokul } \\
(1580 \mathrm{~m})\end{array}$ & Paläotalboden Basis & 170 & Glazifluvial & OSL & $42 \pm 4$ & Letztes Glazial & Broz Periode & OWEN et al. (2002)* \\
\hline 3 & $\begin{array}{l}\text { Sheri / Broz } \\
(1520 \mathrm{~m})\end{array}$ & $\begin{array}{l}\text { Terrasse unter } \\
\text { Schuttkegel }\end{array}$ & 60 & Fluvial & OSL & $41 \pm 6$ & Letztes Glazial & Broz Periode & OWEN et al. (2002)* \\
\hline 4 & $\begin{array}{l}\text { Lawi / Drosh } \\
(1475 \mathrm{~m})\end{array}$ & Glazialer Paläosee & 195 & $\begin{array}{l}\text { Glazio- } \\
\text { lakustrisch }\end{array}$ & OSL & $38 \pm 3$ & Letztes Glazial & Drosh & OWEN et al. (2002)* \\
\hline 5 & $\begin{array}{l}\text { Muli Parwak / Miragram } \\
(2200 \mathrm{~m})\end{array}$ & Grund-/Endmoräne & 95 & Glazifluvial & OSL & $32 \pm 4$ & Letztes Glazial & Drosh / Pret & OWEN et al. (2002)* \\
\hline 6 & $\begin{array}{l}\text { Ausmündung Bumburet- } \\
\text { Tal }(1500 \mathrm{~m})\end{array}$ & Terrasse & 120 & Fluvial & OSL & $22 \pm 3$ & Letztes Glazial & Ayun Periode & OWEN et al. (2002)* \\
\hline 7 & $\begin{array}{l}\text { Gurchako/ Ayun } \\
(1580 \mathrm{~m})\end{array}$ & Paläotalboden Basis & 195 & Glacifluvial & OSL & $17,5-33,8$ & Letztes Glazial & Ayun Periode & OWEN et al. (2002)* \\
\hline \multirow[t]{2}{*}{8} & $\begin{array}{l}\text { Turi Parwak } \\
(2200 \mathrm{~m})\end{array}$ & Flußufer & 30 & $\begin{array}{l}\text { Glazi- } \\
\text { lakustrisch }\end{array}$ & OSL & $8 \pm 1$ & Frühes Holozän & ---- & OWEN et al. (2002)* \\
\hline & Swat, Kohistan & & & & & & & & \\
\hline 9 & $\begin{array}{l}\text { Kalam } \\
(2200 \mathrm{~m})\end{array}$ & Stauseeterrasse & $-\cdots$ & $\begin{array}{l}\text { Glazi- } \\
\text { lakustrisch }\end{array}$ & OSL & $77 \pm 18$ & Letztes Glazial & Gabral II & RICHARDS et al. (2000) \\
\hline 10 & $\begin{array}{l}\text { Kalam } \\
(2200 \mathrm{~m})\end{array}$ & Endmoräne & ---- & $\begin{array}{l}\text { Glazi- } \\
\text { lakustrisch }\end{array}$ & OSL & $38 \pm 10$ & Letztes Glazial & Kalam I & RICHARDS et al. (2000) \\
\hline 11 & $\begin{array}{l}\text { Kalam } \\
(2200 \mathrm{~m})\end{array}$ & $\begin{array}{l}\text { Lößdecke Gabral-II- } \\
\text { Terrasse }\end{array}$ & --- & Löß & TL & $22 \pm 2$ & Letztes Glazial & Gabral & OWEN et al. (1992) \\
\hline \multirow[t]{2}{*}{12} & $\begin{array}{l}\text { Kalam } \\
(2200 \mathrm{~m})\end{array}$ & $\begin{array}{l}\text { Lößdecke Kalam-I- } \\
\text { Terrasse }\end{array}$ & ---- & Löß & $\mathrm{TL}$ & $7 \pm 1$ & Neoglazial & Kalam & OWEN et al. (1992) \\
\hline & Hunza, Karakorum & & & & & & & & \\
\hline 13 & Hunza-/Gulkhin-Tal & Stauseesedimente & ---- & $\begin{array}{l}\text { Glazi- } \\
\text { lakustrisch }\end{array}$ & $\mathrm{TL}$ & $139 \pm 13$ & Mittleres Glazial & Yunz & DERBYSHIRE et al. (1984) \\
\hline 14 & Hunza-/Pasu-Tal & Stauseesedimente & --- & $\begin{array}{l}\text { Glazi- } \\
\text { lakustrisch }\end{array}$ & $\mathrm{TL}$ & $65 \pm 0$ & Letztes Glazial & $\begin{array}{l}\text { Borit Jheel/ } \\
\text { Gulkhin I }\end{array}$ & DERBYSHIRE et al. (1984) \\
\hline 15 & Hunza-/Batura-Tal & Grundmoräne & ---- & $\begin{array}{l}\text { Glazi- } \\
\text { lakustrisch }\end{array}$ & $\mathrm{TL}$ & $50 \pm 3$ & Letztes Glazial & Gulkhin I & DERBYSHIRE et al. (1984) \\
\hline 16 & Unterhalb Pisan-Gletscher & Moräne & ---- & $\begin{array}{l}\text { Glazi- } \\
\text { lakustrisch }\end{array}$ & $\mathrm{TL}$ & $47 \pm 2$ & Letztes Glazial & Gulkhin I & DERBYSHIRE et al. (1984) \\
\hline 17 & $\begin{array}{l}\text { Zusammenfluß Gilgit und } \\
\text { Hunza }\end{array}$ & --- & --- & $\begin{array}{l}\text { Glazi- } \\
\text { lakustrisch }\end{array}$ & $\mathrm{TL}$ & $31 \pm 2$ & Letztes Glazial & ---- & SHRODER et al. (1989) \\
\hline
\end{tabular}




\begin{tabular}{|c|c|c|c|c|c|c|c|c|c|}
\hline 18 & Minapin-Gletscher & $\begin{array}{l}\text { Seitenmoräne, } \\
\text { Außenflanke }\end{array}$ & $\cdots$ & Juniperus & ${ }^{14} \mathrm{C}$ & $0,830 \pm 0,1$ & Little Ice Age & Pasu I & DERBYSHIRE et al. (1984) \\
\hline \multirow[t]{2}{*}{19} & Minapin-Gletscher & $\begin{array}{l}\text { Seitenmoräne, } \\
\text { Innenflanke }\end{array}$ & ---- & Holz & ${ }^{14} \mathrm{C}$ & $0,325 \pm 0,1$ & Little Ice Age & Pasu I & DERBYSHIRE et al. (1984) \\
\hline & \multicolumn{9}{|l|}{ Nanga Parbat, Himalaya } \\
\hline 20 & Raikot-Tal & Grundmoräne & 1000 & $\begin{array}{l}\text { Quarz aus } \\
\text { Erratikum }\end{array}$ & ${ }^{10} \mathrm{Be}$ & $56 \pm 5$ & Letztes Glazial & --- & PHILLIPS et al. (2000) \\
\hline 21 & $\begin{array}{l}\text { Indus-Tal, westlich von } \\
\text { Gor }\end{array}$ & Moräne & --- & $\begin{array}{l}\text { Quarz aus } \\
\text { Erratikum }\end{array}$ & ${ }^{10} \mathrm{Be}$ & $54 \pm 5$ & Letztes Glazial & --- & PHILLIPS et al. (2000) \\
\hline 22 & Oberes Astor-Tal & Glazialer Paläosee & --- & $\begin{array}{l}\text { Glaci- } \\
\text { lakustrisch }\end{array}$ & IRSL & $34 \pm 5$ & Letztes Glazial & --- & PHILLIPS et al. (2000) \\
\hline 23 & $\begin{array}{l}\text { Indus-Tal, westlich von } \\
\text { Gor }\end{array}$ & Moräne & --- & $\begin{array}{l}\text { Quarz aus } \\
\text { Erratikum }\end{array}$ & ${ }^{10} \mathrm{Be}$ & $34 \pm 3$ & Letztes Glazial & $-\cdots$ & PHILLIPS et al. (2000) \\
\hline 24 & $\begin{array}{l}\text { Liachar } \\
(1330 \mathrm{~m})\end{array}$ & Liachar Verwerfung & --- & Glaziofluvial & OSL & $23 \pm 4$ & Letztes Glazial & ---- & RICHARDS et al. (2000) \\
\hline 25 & $\begin{array}{l}\text { Tarishing, Rupal-Tal } \\
(2600 \mathrm{~m})\end{array}$ & Eiskontaktfächer & $-\cdots$ & Glazifluvial & OSL & $15 \pm 4$ & Letztes Glazial & ---- & RICHARDS et al. (2000) \\
\hline 26 & Rupal-Tal & --- & --- & $\begin{array}{l}\text { Quarz aus } \\
\text { Erratikum }\end{array}$ & ${ }^{10} \mathrm{Be}$ & $8,4 \pm 1$ & Holozän & ---- & PHILLIPS et al. (2000) \\
\hline 27 & $\begin{array}{l}\text { Indus-Tal, westlich von } \\
\text { Gor }\end{array}$ & Moräne & --- & $\begin{array}{l}\text { Quarz aus } \\
\text { Erratikum }\end{array}$ & ${ }^{10} \mathrm{Be}$ & $8,0 \pm 2$ & Holozän & --- & PHILLIPS et al. (2000) \\
\hline 28 & Buldar-Gletscher & Seitenmoräne & --- & $\begin{array}{l}\text { Quarz aus } \\
\text { Erratikum }\end{array}$ & ${ }^{10} \mathrm{Be}$ & $8,0 \pm 1$ & Holozän & & PHILLIPS et al. (2000) \\
\hline 29 & $\begin{array}{l}\text { Indus-Tal, westlich von } \\
\text { Gor }\end{array}$ & Moräne & --- & $\begin{array}{l}\text { Quarz aus } \\
\text { Erratikum }\end{array}$ & ${ }^{10} \mathrm{Be}$ & $7,8 \pm 2$ & Holozän & --- & PHILLIPS et al. (2000) \\
\hline 30 & Oberes Astor-Tal & Glazialer Paläosee & $\cdots$ & $\begin{array}{l}\text { Glazi- } \\
\text { lakustrisch }\end{array}$ & IRSL & $6,6 \pm 1$ & Holozän & --- & PHILLIPS et al. (2000) \\
\hline 31 & Rupal-Tal & --- & --- & $\begin{array}{l}\text { Quarz aus } \\
\text { Erratikum }\end{array}$ & ${ }^{10} \mathrm{Be}$ & $6,2 \pm 1$ & Holozän & ---- & PHILLIPS et al. (2000) \\
\hline 32 & Raikot-Gletscher & Moräne & --- & $\begin{array}{l}\text { Quarz aus } \\
\text { Erratikum }\end{array}$ & ${ }^{10} \mathrm{Be}$ & $5,4 \pm 1$ & Holozän & --- & PHILLIPS et al. (2000) \\
\hline 33 & Raikot-Gletscher & Moräne & --- & $\begin{array}{l}\text { Quarz aus } \\
\text { Erratikum }\end{array}$ & ${ }^{10} \mathrm{Be}$ & $5,3 \pm 1$ & Holozän & --- & PHILLIPS et al. (2000) \\
\hline 34 & Rupal-Tal & --- & $-\cdots$ & $\begin{array}{l}\text { Quarz aus } \\
\text { Erratikum }\end{array}$ & ${ }^{10} \mathrm{Be}$ & $4,7 \pm 1$ & Holozän & --- & PHILLIPS et al. (2000) \\
\hline 35 & Rupal-Tal & $\begin{array}{l}\text { Felssturzmaterial } \\
\text { Auswaschungsebene }\end{array}$ & $\cdots$ & $\begin{array}{l}\text { Quarz aus } \\
\text { Erratikum }\end{array}$ & ${ }^{10} \mathrm{Be}$ & $4,1 \pm 1$ & Holozän & --- & PHILLIPS et al. (2000) \\
\hline & Indus-Tal & & & & & & & & \\
\hline 36 & $\begin{array}{l}\text { Shatial } \\
(850 \mathrm{~m})\end{array}$ & Seitenmoräne & 1000 & Lakustrisch & OSL & $59 \pm 9$ & Letztes Glazial & ---- & RICHARDS et al. (2000) \\
\hline 37 & $\begin{array}{l}\text { Shatial } \\
(850 \mathrm{~m})\end{array}$ & Stauseeterrasse & 1000 & Lakustrisch & $\mathrm{TL}$ & $56 / 38 \pm 3$ & Letztes Glazial & --- & SHRODER et al. (1989) \\
\hline 38 & Chilas / Ke Gas & $\begin{array}{l}\text { Seitenmoräne } \\
\text { "Jalipur Tillit" }\end{array}$ & $100-350$ & $\begin{array}{l}\text { Glazi- } \\
\text { lakustrisch }\end{array}$ & OSL & $27 \pm 4$ & Letztes Glazial & --- & RICHARDS et al. (2000) \\
\hline
\end{tabular}

+ Werte gerundet.

* OWEN et al. (2002) datierten die von KAMP (1999) gelieferten Alter neu.

\# Dieses Alter konnte von OWEN et al. (2002) nicht erfolgreich neu datiert werden. 


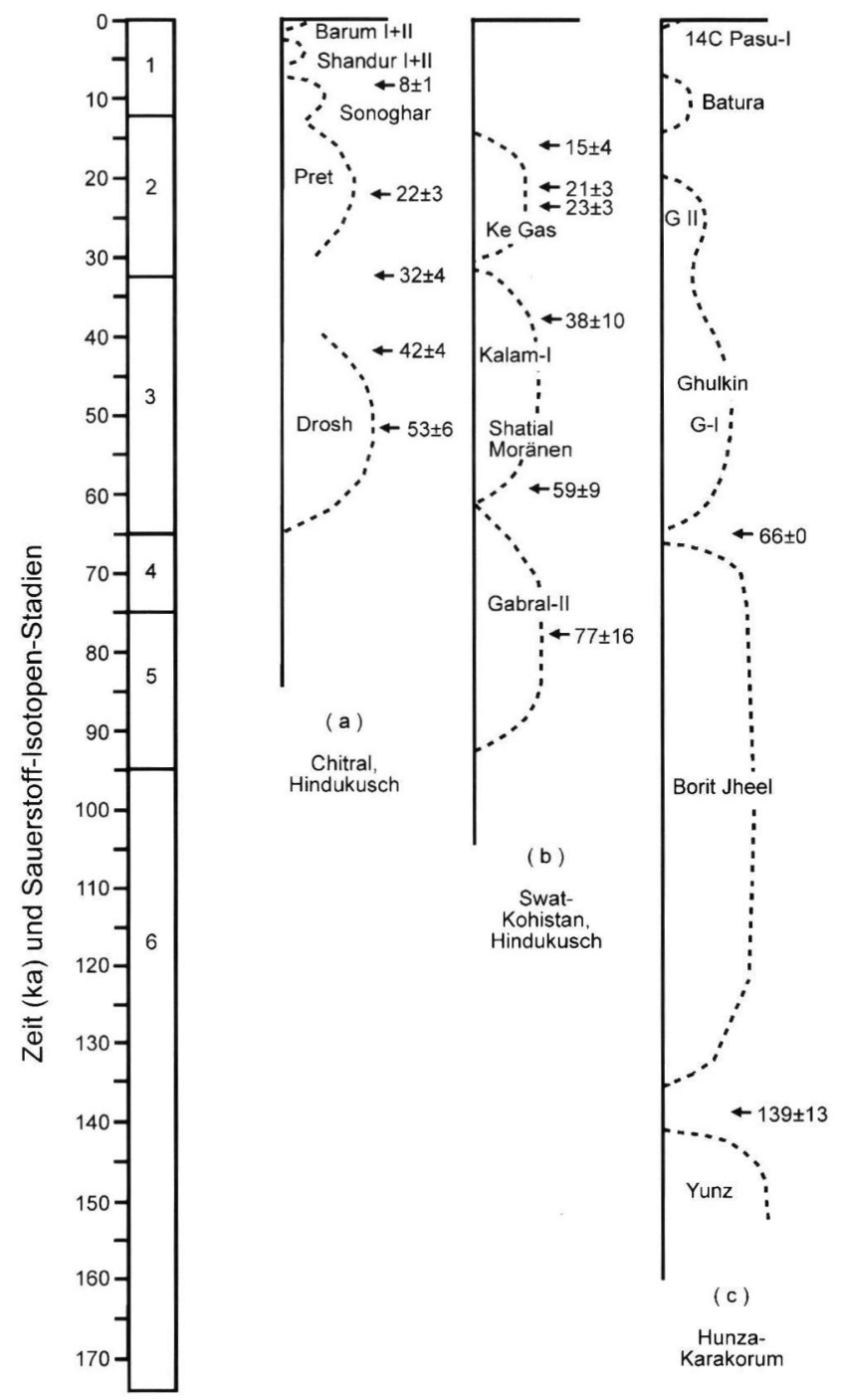

Abb. 4: Chronologien und Daten der quartären Gletscherhochstände und -minima für verschiedene Regionen Nordpakistans (nach KAMP 1999, verändert): (a) KAMP (1999; Alter korrigiert nach OwEN et al., 2002), (b) Richards et al. (2000), (c) DERBYSHIRE et al. (1984).

Fig. 4: Chronologies and datings of Quaternary glacier maxima and minima for different regions in northern Pakistan (after KAMP 1999, revised): (a) KAMP (1999; ages corrected by OwEN et al., 2002), (b) Richards et al. (2000), (c) Derbyshire et al. (1984).

ein Einsetzen ab ca. 18 ky BP; im Falle des rLGM ein Einsetzen nach dem rLGM.

Zeiteinteilungen: neoglazial $=$ Beginn Holozän bis historisch; historisch $=1000 \mathrm{BP}$ bis rezent; rezent $=20$. Jh. bis aktuell.

\section{Hindukusch und Kohistan}

\section{1 Chitral}

Während Karakorum und Himalaya schon relativ früh und z. T. ausgiebig glazialmorphologisch untersucht wurden (s. Kap. 4 u. 5), lagen intensivere Studien für den pakistanischen Hindukusch lange nicht vor. Selbst der westliche Hindukusch in Afghanistan war hinsichtlich seiner pleistozänen Landschaftsgeschichte lange besser bekannt (DESIO 1975; GRÖTZBACH \& RathJENS 1969; Mirwald \& RoEMER 1967). Erste Hinweise auf quartäre Ablagerungen gaben HaSERODT $(1968,1982)$ sowie Buchroithner (1985) in seiner geologischen Karte des Tirich-Mir-Gebietes. HASERODT (1989a u. b) lieferte dann erste ausführliche Beschreibungen zur pleistozänen Vergangenheit der Talschaft Chitral. Seine umfassende Karte der „,Gegenwärtigen und jungpleistozänen [= letztglazialen] Vergletscherung zwischen Hindukusch und Karakorum" (Abb. 2), die als eine vorläufige, weiteren detaillierteren Forschungen dienliche Arbeitsgrundlage angeboten wurde, konnte nun für das Gebiet Chitral durch Kamp (1999), der das erste umfassende Modell der jungquartären Vergletscherungsgeschichte für den östlichen Hindukusch liefert (Tab. 1), verbessert werden (Abb. 3). Dabei zeigte sich, dass die letztglaziale Vergletscherung in Chitral ausgeprägter war, als HASERODT es noch vermutete. OwEN et al. (2002) mussten jedoch die von KaMP (1999, 2001a u. b) gelieferten Datierungen nach der Optisch-Stimulierten-Lumineszenz-Methode (OSL) revidieren und verbessern (Tab. 2). Die Zusammenschau der Arbeiten von KAMP und OwEN et al. zeichnet folgendes verbesserte Modell für die Landschaftsevolution in Chitral (Abb. 4):

Obwohl Relikte eines älteren Glazials nur noch in höher gelegenen Bereichen erhalten sind, kann eine ausgedehnte Vergletscherung angenommen werden. Im nachfolgenden Drosh-Glazial (Sauerstoff-Isotopenstadium 3, OIS-3) war Chitral von einem ausgedehnten Eisstromnetz eingenommen, dessen bedeutend mehr als $500 \mathrm{~m}$ mächtiger Haupttalgletscher ca. $270 \mathrm{~km}$ hinab bis nach Drosh (1300 m ü.M.) reichte, wo die talabwärts letzten glazialen Befunde kartiert werden konnten. 
Tab. 3: Rezente und letztglaziale Schneegrenzen (ELA) und Schneegrenzdepressionen in Nordpakistan (aus KAMP, 1999, verändert).

Tab. 3: Recent and Last Glacial equilibrium lines (ELA) and equilibrium line depressions in the mountains of northern Pakistan (after KAMP, 1999, revised).

\begin{tabular}{|c|c|c|c|c|c|}
\hline Region & $\begin{array}{l}\text { Schneegrenze } \\
\text { (regionales) } \\
\text { Letztglaziales } \\
\text { Maximum } \\
\text { (m üM) }\end{array}$ & $\begin{array}{l}\text { Schneegrenze } \\
\text { Spätglazial } \\
(\mathrm{m} \text { üM) }\end{array}$ & $\begin{array}{c}\text { Rezente } \\
\text { Schneegrenze } \\
\text { (m üM) }\end{array}$ & $\begin{array}{c}\text { Depression } \\
\text { Schneegrenze } \\
\text { (m) }\end{array}$ & Referenz \\
\hline Hindukusch, Chitral & 4050 & ---- & 5050 & 1000 & KAMP (1999) \\
\hline Hindukusch, Chitral & $3700-4000$ & --- & $4800-5200$ & $1100-1200$ & HASERODT (1989b) \\
\hline Hindukusch, Ghizar & --- & $3900-4300$ & $3800-4900$ & $600-1000$ & HASERODT (1989b) \\
\hline Hindukusch & $3000-3600$ & --- & $4000-4600$ & 1000 & PORTER (1970) \\
\hline Kohistan, Swat & $3000-3200$ & --- & $4100-4400$ & 1000 & PORTER (1970) \\
\hline Karakorum, Hunza & 4000 & --- & 5000 & 1000 & PAFFEN (1959) \\
\hline Karakorum, Hunza & ---- & $4200-4300$ & 5000 & $700-800$ & BAtURA GLACIER INVESTIGATION GROUP (1979) \\
\hline Karakorum, Hunza & --- & ---- & 5000 & --- & SHI YAFENG \& ZHANG XIANGSONG (1984) \\
\hline Karakorum, Hunza & $-\cdots$ & --- & $4800-5200$ & --- & VISSER (1928) \\
\hline Karakorum, Rakaposhi & $-\cdots$ & --- & $4500-5400$ & --- & SCHNEIDER (1969) \\
\hline Karakorum & $-\ldots$ & --- & -..- & 1000 & XU DAOMING (1991) \\
\hline Karakorum & --.- & --- & --- & 950 & OWEN (1988) \\
\hline Himalaya, Nanga Parbat & $3000-3700$ & --- & $3750-5200$ & $600-1200$ & SCOTT (1992) \\
\hline Himalaya, Nanga Parbat & --- & --- & ---- & 1200 & KuHLE $(1996 \mathrm{a}+\mathrm{b})$ \\
\hline Himalaya, Nanga Parbat & ---- & ---- & ---- & 1100 & OWEN (1988) \\
\hline Himalaya, Kaghan-Gebiet & $3500-3600$ & --- & 4600 & $1000-1100$ & HASERODT (1989b) \\
\hline Himalaya, Kashmir & $3200-3500$ & $-\cdots$ & $3900-4300$ & $700-800$ & HOLMES \& STREET-PERROTT (1989) \\
\hline
\end{tabular}

Die Schneegrenze lag bei 3850 m ü.M., was einer Schneegrenzdepression von ca. $1200 \mathrm{~m}$ gegenüber der aktuellen entspricht (die Berechnung erfolgte hier nach einer leicht modifizierten Methode nach Kamp (1999) in Anlehnung an die Gipfelmethode von Louis (1955)). Die Gletscher überformten sowohl das Haupttal als auch die Nebentäler intensiv trogartig und schliffen ihre Flanken. Diese Phase muss als regionale Maximalausdehnung (,,regionales LGM") bezeichnet werden. Noch in der Deglaziationsphase, d. h. bei kaltem Klima, wurden in der Broz-Periode mächtige paraglaziale Fächer von den Seitenbereichen geschüttet, die oftmals das Tal vollständig verbauten und Seen aufstauten. Mit zunehmender Erwärmung nahmen die Niederschläge und Abflüsse zu und zerschnitten die paraglazialen Fächer zur Oberterrasse. In der anschließenden Ayun-Periode wurden hauptsächlich fluviale Sedimente geschüttet. Auch diese Ablagerungen wurden zerschnitten - zur Mittelterrasse. Eine erneute Klimaverschlechterung leitete das Pret-Glazial (OIS-2/Frühes Holozän) ein, während dessen sich allerdings kein Eisstromnetz mehr ausbilden konnte, auch wenn der bis nach Pret (1670 m ü.M.) reichende Haupttalgletscher von zahlreichen Seitentalgletschern genährt wurde und immerhin noch eine Länge von $200 \mathrm{~km}$ erreichte. Die Schneegrenzdepression dieser Phase betrug ca. $1000 \mathrm{~m}$ (Tab. 3). In den Seitentalmündungen liegende markante jüngere Moränen dokumentieren das spätglaziale bzw. frühholozäne Sonoghar-Stadial. In den eisfreien Talabschnitten hatte bereits zuvor die Schüttung von Sedimen- ten - vornehmlich alluviale Seitentalfächer - der Urghuch-Periode eingesetzt, die anschließend zur Niederterrasse zerschnitten wurden. Im Holozän stießen lokale Gletscher noch in zwei mittelholozänen Shandur-Ständen (I, II) und zur kleinen Eiszeit (Little Ice Age, LIA) im Barum-Stand I vor. Der Barum-Stand II repräsentiert die rezenten Gletscherstände.

Sowohl Haserodt (1989a u. b) als auch KAMP (1999) machten hierüber hinaus auch zahlreiche Aussagen über die glazialmorphologischen Verhältnisse der Nebentäler. So beschrieben sie u. a. vielerorts Trogtäler und Moränen im Barum- und Shishi-Tal sowie im Lutkho-Tal bei Garam Chashma.

\section{2 Swat}

Im südlich an den Chitral-Distrikt angrenzenden Swat-Kohistan konnte PORTER (1970) drei unterschiedliche Hauptvergletscherungen ausgrenzen (Tab. 1, Abb. 5): Ein frühes Laikot-Glazial ist durch Trogtäler und stark verwitterte, hochliegende Moränen belegt. Ein mittleres Gabral-Glazial läßt sich aufgrund zweier Terrassenniveaus in die Stadiale I und II unterteilen. Der Haupttalgletscher aus dem letzten Kalam-Glazial endete mit mehreren Moränenbögen bei Kalam-Ort (2200 m ü.M.). Dieses letzte Glazial kann seinerseits mittels unterschiedlicher Terrassenniveaus in drei Stadiale (Kalam I-III) untergliedert werden. Im Holozän erfolgten mehrere lokale Gletschervorstöße. HA- 


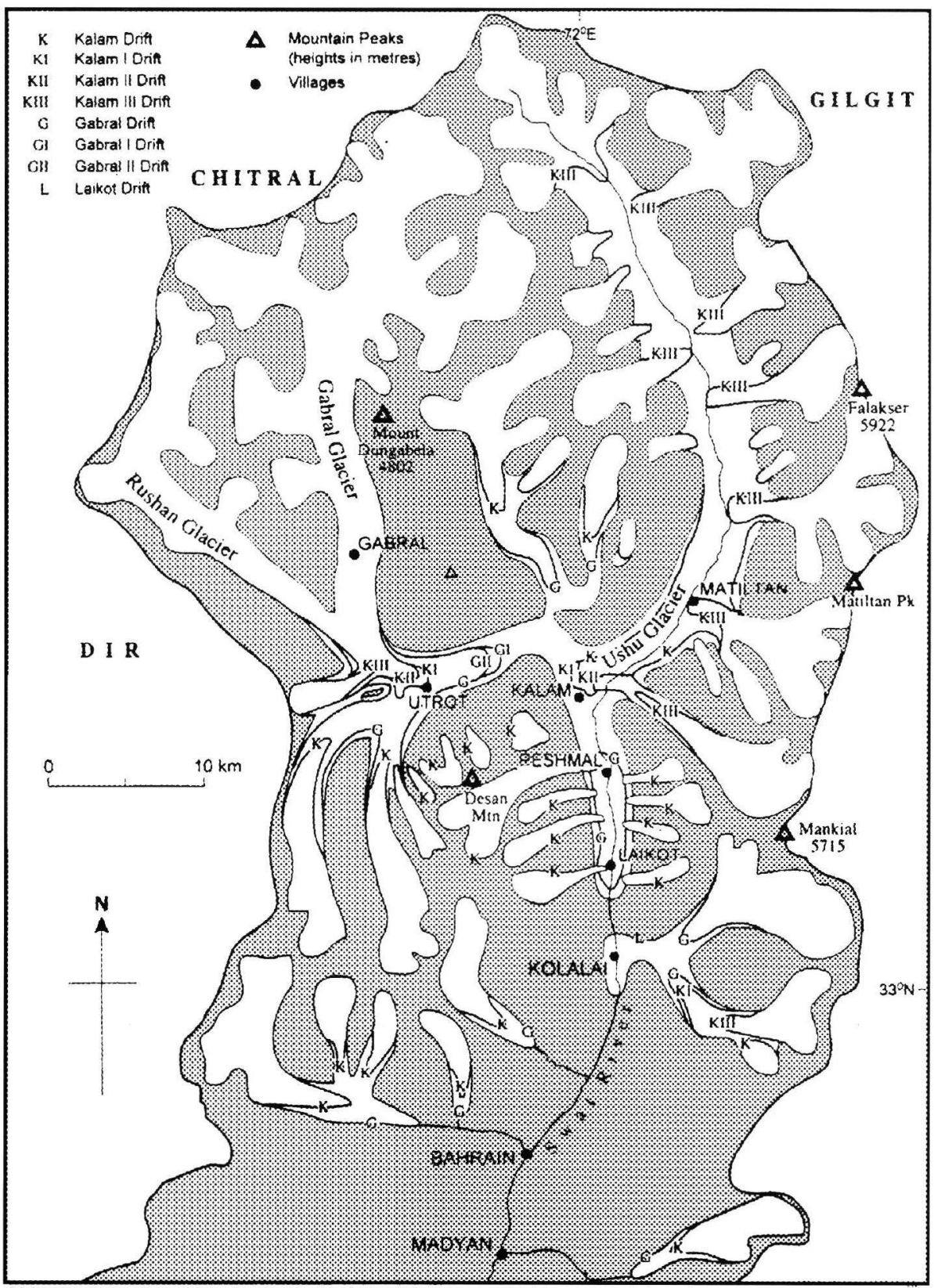

Abb. 5: Ehemalige Gletscherhochstände in Ober-Swat, Kohistan-Hindukusch nach PORTER (1970, verändert): frühes (Laikot), mittleres (Gabral) und letztes (Kalam) Glazial.

Fig. 5: Former glacial maxima in Upper Swat, Kohistan-Hindu Kush after PoRTeR (1970, revised): early (Laikot), middle (Gabral) and last (Kalam) glacial stage.

SERODT (1989b) stellte die Moränen von Kalam und diejenigen von Battakundi im Kaghan-Gebiet (s. Kap. 4.2) in eine Phase.

OwEN et al. (1992) datierten die Lößdecken auf den Terrassen mittels der ThermolumineszenzMethode (TL). Die insgesamt eher jungen Alter sind auf Resedimentierungen zurückzuführen, dennoch geben sie Mindestalter der unterlagernden Terrassen an. So ist der auf der Gabral-Ter- 
rasse liegende Schwemmlöß um $20 \mathrm{ky}$ alt, der auf der Kalam-Terrasse liegende um 7 ky alt (Tab. 2). Die Autoren stellten daher das Laikot-Stadial in das frühe Letztglazial, das Gabral-Stadial in die Phase des LGM und das Kalam-Stadial in das Neoglazial.

Untersuchungen von RicharDs et al. (2000) lieferten die neuesten absoluten OSL-Daten von verschiedenen Sedimenten (Tab. 2, Abb. 4): Das Gabral-II-Stadial hat ein Alter von 77 ky und wäre demnach auch hier in das Letztglazial zu stellen. Das Kalam-I-Stadial datiert 38 ky und repräsentiert eine Vorstoßphase während des jüngeren Letztglazials.

Aus allen Untersuchungen ergibt sich das Bild einer ausgeprägten letztglazialen Vergletscherung mit vier Stadialen und einem im globalen Vergleich frühen „regionalen LGM”.

\section{3 Ghizar-Gebiet}

Für das Ghizar-Gebiet zwischen dem nach Chitral verbindenden Shandur-Paß im Westen und Gilgit im Osten liegen nach wie vor nur wenige Aussagen zur eiszeitlichen Vergletscherung vor. WiCHE (1958) lieferte Ergebnisse für den sogenannten „Hindu Raj“" und südlich sich anschlieBende Gebiete. In dem Tangir- und dem DarelTal fand er Moränen in $1800 \mathrm{~m}$ bzw. $1470 \mathrm{~m}$ ü.M. nahe der Einmündung in das Indus-Tal.

Erst HASERODT (1989b) versuchte einen ersten größeren Gesamtüberblick für das Ghizar-Gebiet (Abb. 2): Ausgehend vom vereisten ShandurDiffluenzpaß (3700 m ü.M.) erstreckte sich ein letztglazialer Gletscher durch das Ghizar-Tal lediglich bis nach unterhalb von Chachi. In dem Gebiet um Chachi (2800-3100 m ü.M.) finden sich zahlreiche Moränen und der auf ein Toteisaustauen zurückgehende Phandar-See (2880 $\mathrm{m}$ ü.M.). Dagegen waren die Seitentäler bis nahe ihrer Einmündungen in das Ghizar-Haupttal von Eis erfüllt, so z. B. die Täler Khokush, Shunji, Serbal, Chachi, Batret und Gupis. Für das Shunji-Tal rekonstruierte HASERODT einen 40 km langen spätglazialen Gletscher und eine Schneegrenze bei $4300 \mathrm{~m}$ ü.M., d. h. $600 \mathrm{~m}$ unterhalb der rezenten (Tab. 3); für das Hochglazial nahm der Autor eine weit ausgedehntere Vergletscherung an. Das Yassin-Tal war im Letztglazial ausgiebig vergletschert, denn nahe der Talausmündung in das GhizarHaupttal sind die Talflanken bis zu $250 \mathrm{~m}$ hoch mit Moräne verkleidet. Im Yassin-Tal selbst lie- gen bei 2450 m ü.M. markante spätglaziale Moränen, die den Seitentälern Asambar und Qurkulti entstammen und mit den Sonoghar-Moränen in Chitral vergleichbar sind (s. Kap. 3.1). Während dieses Rückzugsstadiums war dieser AsambarQurkulti-Seitentalgletscher $18 \mathrm{~km}$ lang. Die Schneegrenze konnte auf $3950 \mathrm{~m}$ ü.M. berechnet werden, was einer Depression von 800-900 m gegenüber dem heutigen Wert entspricht (Tab. 3). Für die im „Hindu Raj“ wurzelnden, in das Ghizar-Haupttal mündenden Täler befand sich die letztglaziale Schneegrenze bei 3900 m ü.M., d. h. $1000 \mathrm{~m}$ unterhalb der rezenten (Tab. 3). Erst bei der Einmündung des Ishkoman-Tals nordwestlich von Singal im Bereich von Gakuch (19502150 m ü.M.) finden sich wieder Moränen im ab hier nun so heißenden Gilgit-Haupttal. Diese entstammen jedoch einem Ishkoman-Gletscher, der - sogar in das Haupttal einschwenkend - bis hierher reichte. Dieser hochletztglaziale Gletscher hatte eine Länge von $110 \mathrm{~km}$.

Im Gegensatz zu HaSERodt (1989b) hielten DerBYSHIRE \& OWEN (1990) und OWEN $(1988,1989)$ das Gilgit-Tal auch oberhalb der Hunza-Einmündung für vergletschert, und zwar sowohl im mittleren Yunz-Glazial als auch im frühen Borit-JheelStadial des Letztglazials (Abb. 6) (s. Kap. 4.1).

\section{Karakorum}

Der Karakorum kann heute als besterforschter Hochgebirgsraum Nordpakistans gelten. Glazialmorphologische und glaziologische Studien haben hier eine lange Tradition (Dainelui 1922; Haserodt 1984, 1989b; HewITt 1961; Kick 1985; Kuhle 1989; Meiners 1995, 1997; Mercer 1975; Owen et al. 1998; Owen \& Derbyshire 1989; PAFFEN et al. 1956; PILLEWIZER 1962; SCHNEIDER 1959, 1969; SeArle 1991; Shroder 1993; Shroder et al. 1993; von Wissmann 1959; Zhang Xiangsong \& SHI YAFENG 1980). Insbesondere im Rahmen zweier großangelegter Forschungsprogramme, der Batura Glacier Investigation. Group (1976, 1979) und des pakistanisch-chinesisch-britischen International Karakoram Project (DERBYShIRE 1984; Derbyshire et al. 1984; GoudiE et al. 1984; MiLLER 1984; SHI Yafeng \& Zhang Xiangsong 1984; Zhang XIANGSONG 1984) konnten wertvolle Ergebnisse zur Vergletscherung des Karakorums erarbeitet werden. 


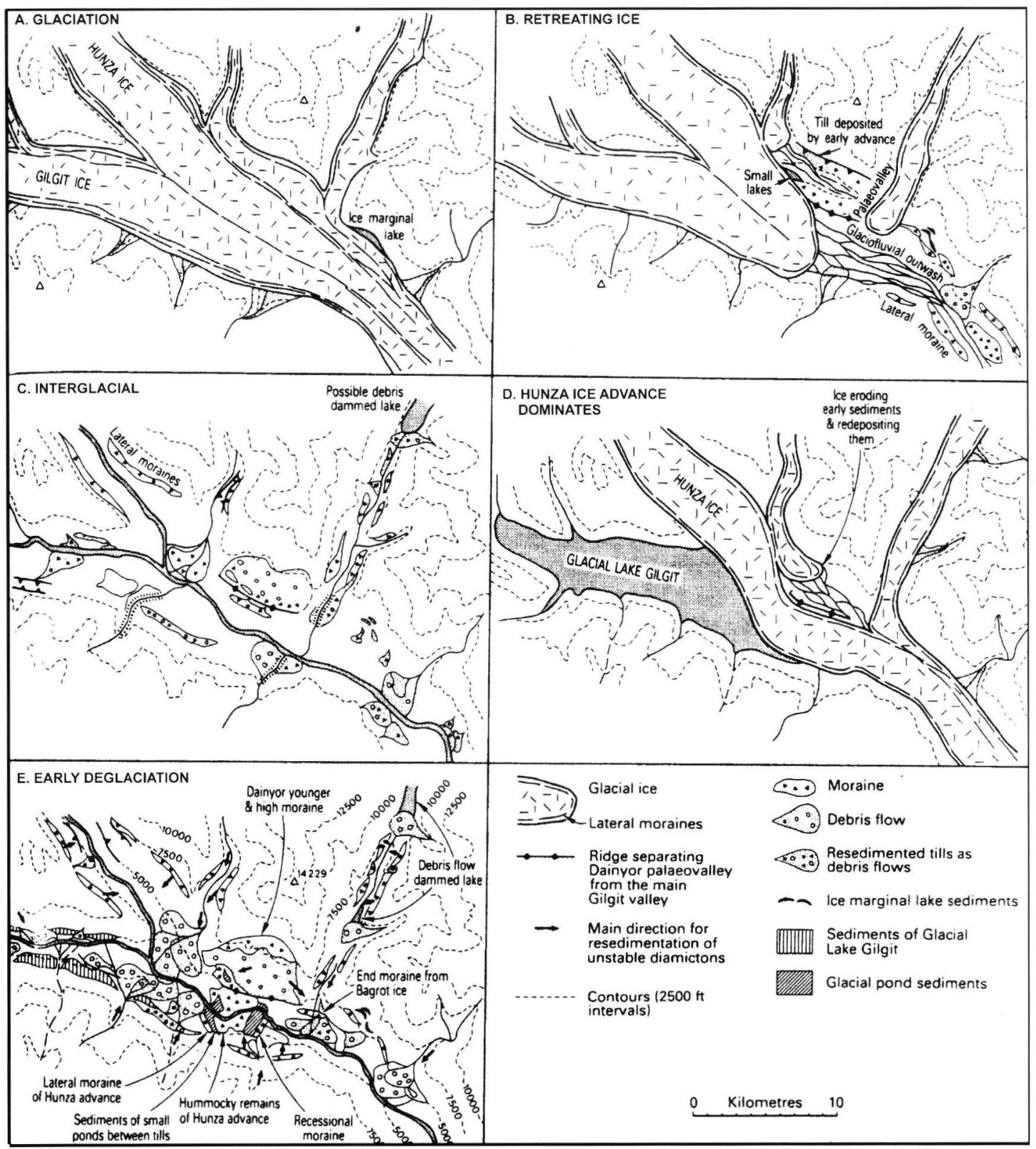

Abb. 6: Gletschervorstöße und -rückzüge in der Konfluenz von Hunza- und Gilgit-Fluß, Gilgit-Gebiet nach OweN (1989, verändert): A - mittlere (Yunz) Vergletscherung, D - letzte (Borit Jheel) Vergletscherung.

Fig. 6: Glacier advances and retreats in the confluence of Hunza and Gilgit rivers, Gilgit area after OwEN (1989, revised): A middle (Yunz) glaciation, D - last (Borit Jheel) glaciation.

\section{1 Hunza und Gilgit-Gebiet}

Erste detaillierte Arbeiten im Hunza-Karakorum gehen zurück auf PAFFEN et al. (1956) und SCHNEIDER (1959). Beide Autoren entwickelten eine Vorstellung von drei Vergletscherungen. SCHNEIDER kam für den Batura-Gletscher zu der Vorstellung, dass dieser im Pleistozän maximal bis nach Saret in Mittel-Hunza gereicht hat und somit $30 \mathrm{~km}$ lang war; der damals $8 \mathrm{~km}$ lange Minapin-Gletscher reichte bis nach Hindi. SCHNEIDER sowie PAFFEN et al. und auch HASERODT (1989b) verneinten einen durchgehenden letztglazialen Hunza-Haupt- 

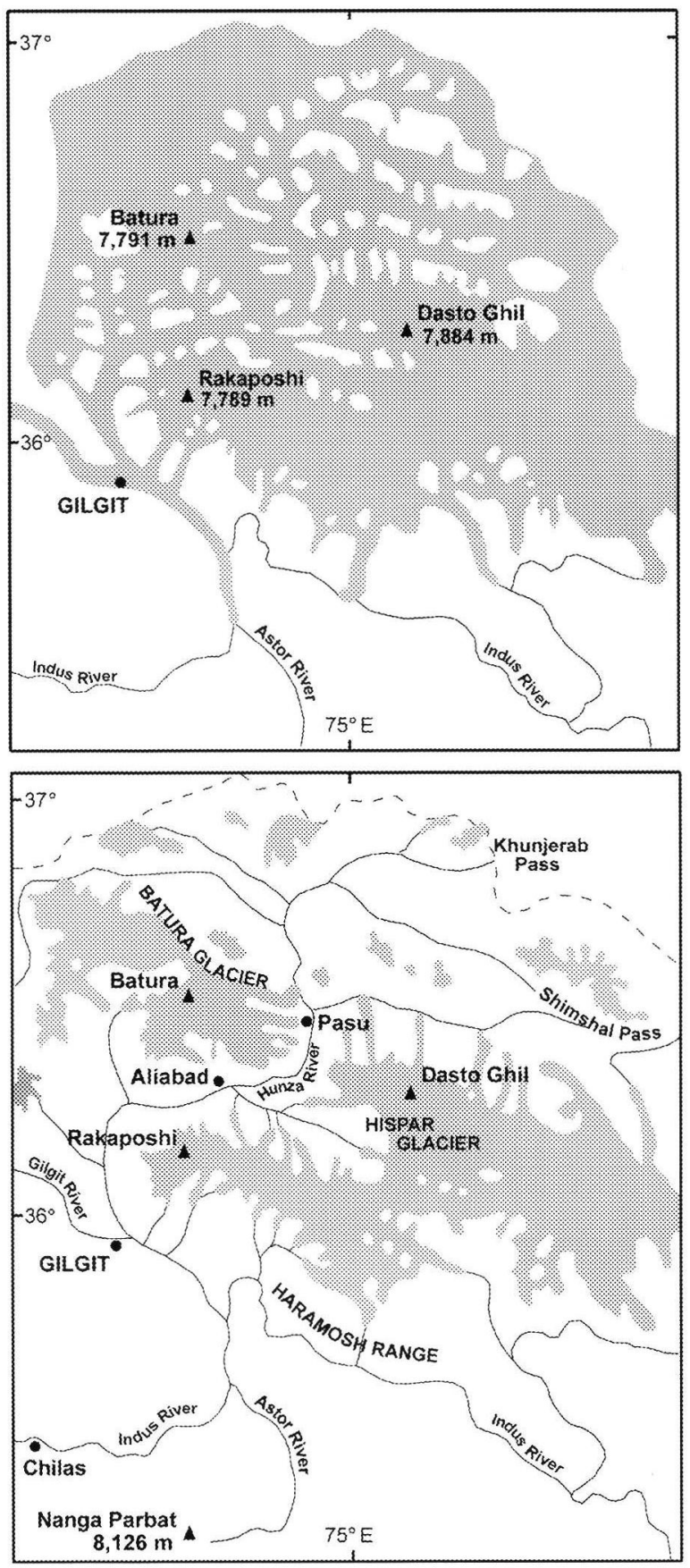

Abb. 7: Heutige Gletscher und letztes (Borit Jheel) Glazial (s. o.) im Hunza-Karakorum nach Derbyshire et al. (1984, Neuzeichnung).

Fig. 7: Present glaciation and last (Borit Jheel) glaciation (s. above) in the Hunza-Karakoram after Derbyshire et al. (1984, new drawing). talgletscher bis hinab nach Gilgit. Vielmehr interpretierten sie die z. B. bei Chalt, Nager und Khaibar (2680 $m$ ü.M.) liegenden Moränenwälle als Hinterlassenschaften ehemaliger Seitentalgletscher, wobei PAFFEN et al. diese ins Spätglazial stellten und eine Schneegrenzdepression von $1000 \mathrm{~m}$ an (Tab. 3) annahmen; HASERODT sah sie als Maximalstand des Letztglazials an. Letzterer Autor machte weitere Ausführungen zu der Vergletscherung in diesem Raum (Abb. 2): Der Shimshal-Gletscher in Ober-Hunza z. B. endete bereits im mittleren Seitental und stieß nicht bis in das Hunza-Haupttal vor. Das Becken von Sost (2765 m ü.M.) war ein Sammelbecken für zahlreiche Gletscher, die sich hier vereinigten und noch $8 \mathrm{~km}$ talabwärts reichten. Einen durchgehenden Talgletscher im oberhalb von Sost gelegenen Kunjerab-Tal hielt der Autor für eher unwahrscheinlich, auch wenn der Kunjerab-Paß (4748 m ü.M.) selbst vergletschert gewesen sein muß. Im nordwestlich von Gilgit gelegenen Naltar-Tal endete der spätglaziale Gletscher $30 \mathrm{~km}$ talabwärts des heutigen Gletschers in $2300 \mathrm{~m}$ ü.M.. Für das Spätglazial nannte die Batura Glacier InvESTIGATION Group (1979) am Batura-Gletscher eine Schneegrenzdepression von $700-800 \mathrm{~m}$ gegenüber der rezenten Schneegrenze, die im Mittel bei $5000 \mathrm{~m}$ ü.M. liegt. Den gleichen Wert für die rezente Schneegrenze bestimmten auch SHI YAFENG \& ZHANG XiAngsong (1984), während VISSER (1928) mit 48005200 m ü.M. genauere Angaben machte.

Dieser Auffassung einer weniger intensiven Vergletscherung widersprachen DERBYSHIRE et al. (1984), die für Hunza drei intensive Glaziale rekonstruierten, wobei für das Letztglazial drei Stadiale ausgewiesen wurden (Tab. 1, Abb. 4). Ein frühes Glazial (Shanoz) war durch eine ausgedehnte Vergletscherung geprägt. Das folgende mittlere Glazial (Yunz) wurde auf $>139 \mathrm{ky}$ datiert (Tab. 2). Das Letztglazial ist zweigeteilt: Seine maximale Ausdehnung hat der Hunza-Gilgit-Indus-Talgletscher in einem frühen Stadial (Borit-Jheel, $65 \mathrm{ky}$ ) erreicht und war bis auf $1300 \mathrm{~m}$ ü.M. hinab zur Astor-Taleinmündung in das IndusTal vorgestoßen (Abb. 7). Im zweiten Stadial (Gulkhin I, < 47 ky) war die Vergletscherung bereits weniger ausgedehnt. Das Spätglazial (Ghulkin II) war schließlich geprägt von Vorstößen der Seitentalgletscher in ein eisfreies 
Gilgit-Indus-Tal, wobei letzteres oft blockiert wurde, und sich infolgedessen Seen aufstauten. Für das Holozän stellten die Autoren einen neoglazialen Stand (Batura), einen LIA-Stand (Pasu I) sowie einen rezenten Stand (Pasu II) fest.

OWen (1989), SHroder (1984) und SHroder et al. $(1989,1993)$ griffen diese glaziale Chronologie auf (Tab. 1). Sie rekonstruierten einen Batura-Gletscher, der im frühen Glazial (Shanoz) $250 \mathrm{~km}$ weit durch das Hunza- und Gilgit-Tal bis Chilas, also weit in das Indus-Tal hinab, reichte. Im Gegensatz zu Derbyshire et al. (1984) sahen sie den vorletztglazialen Indus-Talgletscher (Yunz) jedoch noch weiter talabwärts bis Shatial ( $850 \mathrm{~m}$ ü.M.) reichen (s. Kap. 6), womit dieses mittlere Glazial die ausgedehnteste Vergletscherung repräsentiert. OWEN (1988) lieferte zusätzlich eine Vorstellung von der Vergletscherung im Raum Gilgit (Abb. 6): Während sich Hunza- und Gilgit-Gletscher hier im mittleren Yunz-Glazial vereinigten, reichte im Borit-Jheel-Stadial lediglich der Hunza-Gletscher hinab, der das Gilgit-Tal blockierte und einen See aufstaute. Alle hier genannten Autoren stellten darüber hinaus die beiden Gulkhin-Stadiale ins
Holozän, wobei das Gulkhin-II-Stadial einen Stillstand des Gulkhin-I-Stadials darstellt.

Richards et al. (2000) schließlich verbesserten diese Chronologie aufgund ihrer OSL-Daten (Tab. 2): Der bisher als mittleres Glazial bestimmte YunzStand, dessen Gletscher bis hinab nach Shatial reichte, hat demnach ein Alter von $60 \mathrm{ky}$ und muß daher als „regionales LGM“ angesehen werden. Des weiteren repräsentiert das bislang als rLGM interpretierte Borit-Jheel-Stadial mit 27 ky ein regionales spätglaziales Stadial, währenddessen der Indus-Gletscher in Chilas endete.

Holozäne Gletscherstände wurden von HASERODT (1989b) beschrieben. So kann die Große LateralMoräne („GLM“ nach HASERODT 1984) des Mulungutti-Gletschers in Shim Shal als historische Form mit neoglazialen Anteilen angesehen werden. Morphologisch ähnliche GLM wurden für andere Gebiete auf ca. 280 Jahre vor heute datiert (s. Kap. 4.2). Ein allgemeiner Gletscherrückgang seit etwa 1910-1920 gilt nach Untersuchungen an zahlreichen Gletschern, wie z. B. Biafo, Chogo-Lungma, Hispar, Minapin und Hoppar, mittlerweile als erwiesen (KICK 1985).

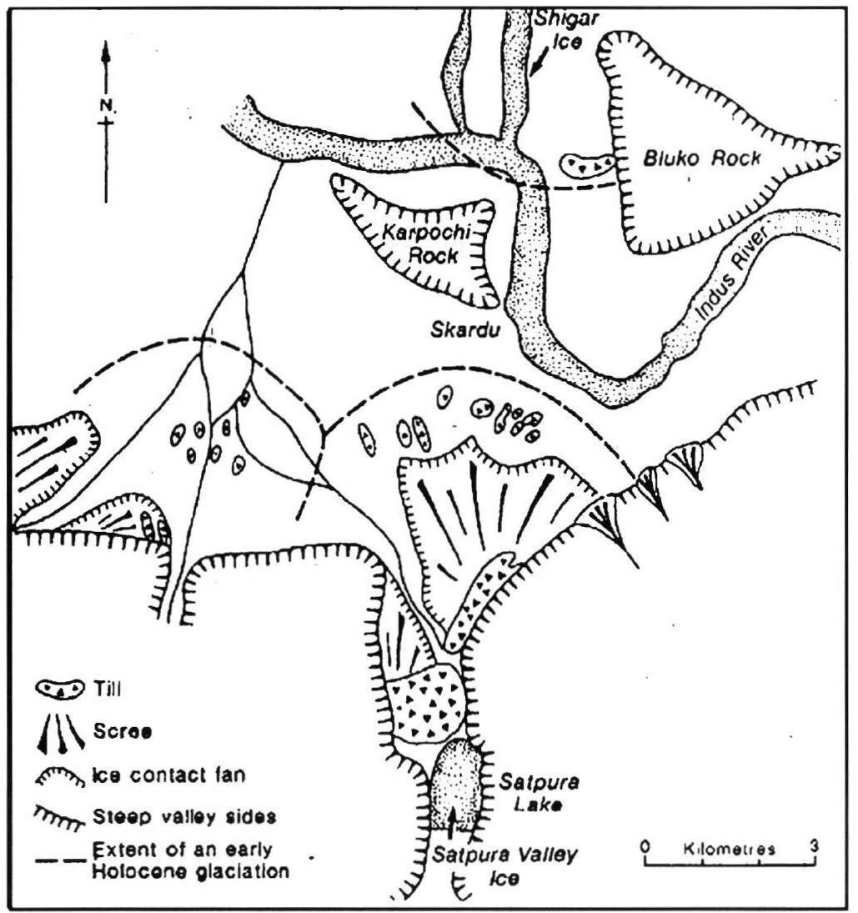

Abb. 8: Frühholozäne Gletschervorstöße im Skardu-Becken, Karakorum, nach OwEN (1988).

Fig. 8: Early Holocene glacier advances in the Skardu basin, Karakoram, after OwEN (1988).

\section{2 Rakaposhi und Haramosh}

Nach Haserodt (1989b) stießen vom Rakaposhi-Massiv im Hochletztglazial zahlreiche Gletscher die Seitentäler hinab (Abb. 2). So querte im Westen der Jaglot-Gletscher sogar das Hunza-Tal bei $1750 \mathrm{~m}$ ü.M. und reichte bis an die gegenüberliegende Talflanke. Ein spätglazialer Gletscherstand ist im Jaglot-Tal noch in $2550 \mathrm{~m}$ ü.M. zu erkennen. Ähnliches gilt für den Bagrot-Gletscher, der im Hochletztglazial mit $25 \mathrm{~km}$ Länge bis nach Oshikandaz (1600 $\mathrm{m}$ ü.M.) in das Gilgit-Tal hinabreichte. Neoglaziale Anteile stellten RöthLISBERGER (1986) sowie RÖTHLISBERGER \& GeYH (1985) in der GLM des Burchee-Gletschers in OberBagrot fest. Die Autoren postulierten deshalb einen markanten Gletschervorstoß zwischen 3600 und 3200 Jahren vor heute. Tieferliegende GLM-Bereiche wurden auf um 585 bzw. 190 Jahre vor heute datiert und entsprechen damit einem historischen Alter. HASERODT (1989b) datierte dieselbe GLM durch Baumringauszählungen auf mindestens 260-290 Jahre vor heute; ein vergleichbares Alter kann für 
die GLM der Gletscher Barpu, Jaglot und Hoppar gelten. Die an die GLM des Burchee-Gletschers innen anlagernden Moränen interpretierte der Autor als Hochstand zwischen 1880 und 1920. SCHNEIDER (1969) gab für das Rakaposhi-Massiv einen rezenten Schneegrenzwert von 4500-5400 m ü.M. je nach Exposition an.

Auch am steilwandigen Haramosh-Massiv nahm HASERODT (1989b) solche Vorstöße an (Abb. 2). Ein vereinigter Mani-Baskei-Khaltoro-Gletscher stieß im Hochletztglazial bis fast in das eisfreie Indus-Tal bei Sassi (1500 m ü.M.) vor, wo markante Seitentalmoränen liegen. Der Mani-Gletscher war dabei $12 \mathrm{~km}$ länger als der rezente. Im Spätglazial reichte dieser Gletscher noch bis auf $2100 \mathrm{~m}$ ü.M. hinab. WicHe (1958) nahm hingegen die pleistozänen Endlagen von Mani- und BaskeiGletscher nur 3-4 km unterhalb der heutigen liegend an. Und während WICHE (1959) die GLM des Mani-Gletschers, die den Kutual-See $(3260$ m ü.M.) umkränzen, noch ins Spätglazial stellte, sah Haserodt (1989b) sie als holozäne Formen an. Gleiches gilt für die GLM des nahen Baskei-Gletschers.

\section{3 Baltistan}

Schon DaInelli (1922) hielt das Skardu-Becken und einige Haupttäler Baltistans für ehemals intensiv und mehrfach vergletschert. OwEN (1988) bemerkte eine Reihe von Planationsoberflächen, wovon er diejenige in Höhen von 5000-5400 m ü.M. als präpleistozän hielt. Haserodt (1989b) beschrieb Moränen auf dem sogenannten Skardu-Berg, die wahrscheinlich einem älteren Glazial entstammen. An der Westflanke des Skardu-Bekkens bei Ponedas wird der Kachura-See (2319 m ü.M.) durch Wallmoränen abgedämmt. Sie wurden im LGM von einem 35 km langen Shigar-Gletscher abgelagert, der in den äußersten NW-Bereich des Skardu-Beckens einschwenkte (Abb. 2). $7 \mathrm{~km}$ südlich von Skardu verbaute ein spätglazialer Seitentalgletscher das damals eisfreie Indus-Tal und staute den Satpara-See auf. Und auch bei Skardu selbst liegen spätglaziale Wallmoränen. HASERODT hielt das Indus-Tal zwischen Skardu und GilgitTaleinmündung für - von Seitentalvorschüben abgesehen - im gLGM weitgehend eisfrei. Moränen solcher tributären Gletscher beschreibt er für Shengus (1800-1950 m ü.M.) und in der Talweitung von Rondu (2050-2150 m ü.M.). Ob dieser Engtalabschnitt des Indus zu jener Zeit jedoch tatsächlich eisfrei gewesen ist, muß heute bezwei- felt werden. Für das Holozän konnte Owen (1988) Gletschervorstöße aus den Seitentälern beschreiben, die im Skardu-Becken endeten (Abb. 8).

\section{4 K2-Region}

In der nördlich des K2 gelegenen KarakorumNordabdachung fand KuHLE $(1989,1998)$ auf mehreren Transfluenzpässen Anzeichen einer ausgedehnten Vergletscherung, so z. B. auf dem Shimshal-Paß oder auf dem das Muztagh- mit dem Shaksgam-Tal verbindenden Paß. Der Autor rekonstruierte eine mindestens $250 \mathrm{~m}$ mächtige Eisdecke auf den Pässen selbst, weshalb er ein ausgedehntes Karakorum-Aghil-Kunlun-Eisstromnetz postulierte.

\section{West-Himalaya}

Wie für den Karakorum kann auch für den pakistanischen West-Himalaya auf eine lange glazialmorphologische und glaziologische Forschungstradition zurückgeblickt werden (BISHOP et al. 1995, 1998; Haserodt 1989b; Finsterwalder 1937; Holmes 1993; Holmes \& Street-Perrot 1989; Kick 1996; Loewe 1924; Oestreich 1906; OweN et al. 1998; Phil.tips et al. 2000; Richards et al. 2000; SHroder 1993; SHRODER et al. 1989, 2000; de Terra 1938; Troll 1938; Wadia 1932/33).

\section{1 Nanga Parbat}

Für den Nanga Parbat stellte ScOтt (1992) zwei Hauptvergletscherungen im mittleren und letzten Glazial, während dessen die Gletscher 15 bzw. $12 \mathrm{~km}$ länger waren als heute, und einen weiteren kleineren Vorstoß im Astor-Tal fest (Abb. 9). Die Autorin verglich dabei die Gletscherstände mit Phasen im Hunza-Karakorum (vgl. Kap. 4.1): Zwei Stillstände im mittleren Astor-Tal ensprechen dem Yunz-Glazial (mittleres Glazial) und dem Borit-Jheel-Stadial (letztes Glazial); ein Stillstand in den unteren Abschnitten der größeren Seitentäler Rupal, Rhakiot und Rama kennzeichnet das Gulkhin-I-Stadial (letztes Glazial); das GulkhinII-Stadial wird durch in diesen Tälern weiter talaufwärts liegende Sedimente belegt; Eiskontaktsedimente nahe den heutigen Gletscherenden datieren in das Batura-Stadial (frühes Holozän); schließlich verkörpert die GLM die historischen Stände Pasu I und II. Während all dieser VorstöBe hat nach SсотT der Astor-Talgletscher niemals bis in das - im gLGM vergletscherte - Indus-Tal hinabgereicht. 


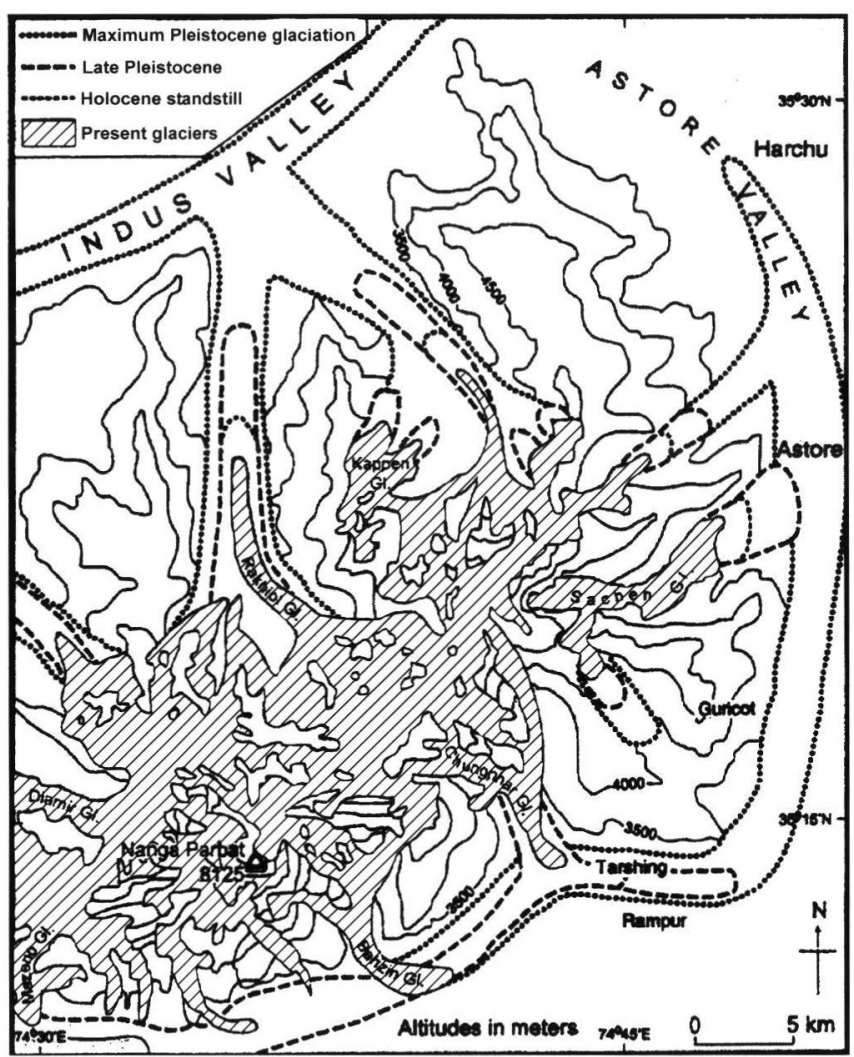

Abb. 9: Ehemalige Gletschervorstöße am Nanga Parbat, Himalaya, nach SCOTT (1992, publiziert in OwEN et al., 2000:78, verändert).

Fig. 9: Former glacier advances at Nanga Parbat, Himalaya, after SCOTT (1992, published in Owen et al., 2000:78, revised).

SLOAN et al. (1998) und PHILLIPS et al. (2000) interpretierten zwei letztglaziale Stadiale, die laut kosmogener ( ${ }^{3} \mathrm{He}$ ) Datierungen um $56 \mathrm{ky}$ und $35 \mathrm{ky}$ stattfanden (Tab. 2). Der letzte Gletscherrückzug aus dem Mündungsbereich des Astors in das Indus-Tal nahe der Lichar-Verwerfung erfolgte nach OSL-Datierungen von RicHARDs et al. (2000) um $27 \mathrm{ky}$ (Tab. 2), womit diese Autoren die letzte große Indus-Haupttalvergletscherung angezeigt sahen. PHILLIPS et al. (2000) argumentierten jedoch dagegen, dass aufgrund der während des OIS-2Stadiums vorherrschenden ariden Klimas ein solcher spätglazialer Gletschervorschub ausgeschlossen sei.

Owen et al. (2000) zeigten, dass im Anschluß an das rLGM noch während des Spätglazials mindestens zwei Gletschervorstöße oder Stillstandsphasen sowie im Holozän mindestens drei begrenzte Vorstöße stattfanden. Auch PhiLlips et al. (2000) beschrieben und datierten mehrere holo- zäne Vorstöße in verschiedenen Seitentälern am Nanga Parbat (Abb. 10, Tab. 2).

Kuhle (1989, 1996a u. b) untersuchte ausführlich das Nanga Parbat Massiv und rekonstruierte ein ausgedehntes Eisstromnetz im letzten Hochglazial. Nach ihm hat die damalige Schneegrenze bei 3400-3700 m ü.M. gelegen, während sie heute bei 4600-5300 $\mathrm{m}$ ü.M. zu finden ist (Tab. 3).

\section{2 Kaghan-Gebiet}

Im Kaghan-Gebiet zog Haserodt (1989b) insbesondere die ausgedehnten Hochtalböden, wie z. B. um Gittidas, als pleistozäne Nährgebiete nach Plateaugletschertyp zur Rekonstruktion der Gletscherausdehnung heran (Abb. 2). Im Kaghan-Kunhar-Tal selbst bei Battakundi (2620 m ü.M.) konnte der Autor Zeugen älterer Glaziale entdecken, die er aufgrund ihrer Lage und Morphologie mit ähnlichen Relikten in Chitral (Haserodt 1989b) und Ober-Hunza (SCHNeider 1959; PAfFEN et al. 1956; Derbyshire et al. 1984) verglich. Sie liegen hier in 2950-3100 m ü.M., d. h. 300-450 m oberhalb der letztglazialen Moränen. Die letztglaziale Eiserfüllung des Tals nahm HASERODT bis in den mittleren Talabschnitt an. Talabwärts bei Kaghan-Ort (2022 m ü.M.) stießen lediglich noch mehrere Seitentalgletscher in das hier eisfreie Haupttal vor. Talaufwärts bei Battakundi (2620 m ü.M.) sind dagegen in der Dadar-Nar-Seitentaleinmündung gestaffelte hoch- bis spätglaziale Wallmoränen erhalten. Zu dieser Zeit hatte der Dadar-Nar-Gletscher somit eine Länge von $16 \mathrm{~km}$. In dem Saif-ul-Muluk-Seitental liegt bei 3214 m ü.M. ein See, der durch spätglaziale Moränen abgedämmt worden ist. HASERODT nahm bei einer rezenten Schneegrenze von $4600 \mathrm{~m}$ ü.M. eine letztglaziale Depression auf 35003600 m ü.M. an (Tab. 3).

\section{3 Deosai-Plateau}

Nach DAINELLI (1922) waren das ausgedehnte Deosai-Plateau und die Deosai-Kette mit ihren 5000$5600 \mathrm{~m}$ hohen Gipfeln gänzlich vergletschert. Aus der Kette reichte ein $25 \mathrm{~km}$ langer Tukshan-Gletscher bis in das nördlich liegende, im Hochletztglazial eisfreie Indus-Tal hinein. Dieser Auffassung schloß sich Haserodt (1989b) an (Abb. 2). 


\section{Indus-Tal}

Seit langer Zeit gibt es eine Kontroverse zu der Frage, ob, wann und wie intensiv das untere und mittlere Indus-Tal vergletschert war, $d$. $h$. also vor allem auch, wie weit ein Haupttalgletscher vordrang. Die Existenz von Moränen und lakustrischen Stauseesedimenten wurde dabei von keinem Autor negiert, doch während die einen das IndusTal selbst im Mittel- und/oder Letztglazial tief hinab und hoch vergletschert sahen, interpretierten die anderen die glazialen Sedimente zumindest für das Letztglazial als von Seitentalgletschern abgelagert. Früh verwarf man allerdings die Vorstellung von TRINKLER (1932) von einem sogar bis ins Gebirgsvorland reichenden Indus-Gletscher; bereits LOEWE (1924) hatte für ein im Letztglazial

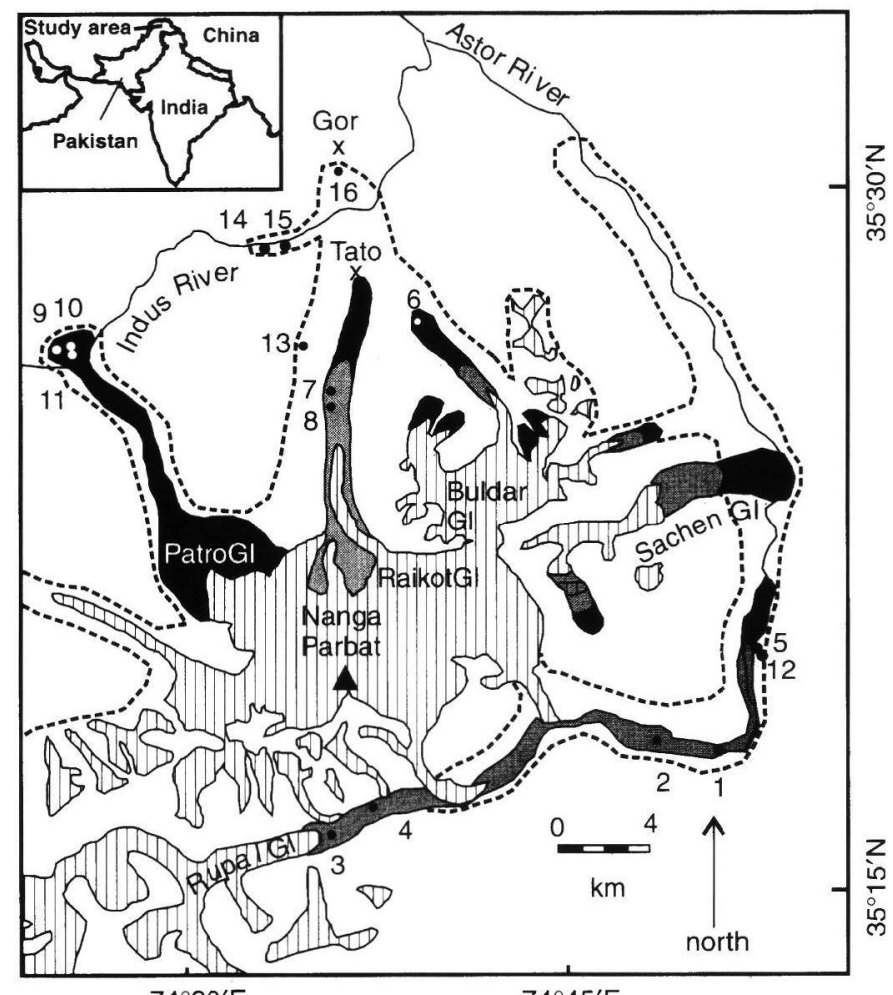

$74^{\circ} 30^{\prime} \mathrm{E}$

$74^{\circ} 45^{\prime} \mathrm{E}$

Abb. 10: Ehemalige Gletschervorstöße am Nanga Parbat, West-Himalaya nach PHILLIPS et al. (2000). Gestreift: Rezente Gletscher; Schwarz: maximale Ausdehung im Holozän; Schattiert: mittelholozäner Vorstoß und/ oder Stillstand; Gestrichelte Linie: maximale Ausdehnung während des Sauerstoff-Isotopen-Stadiums 3 (OIS-3).

Fig. 10: Former glacier advances at Nanga Parbat, West-Himalayas after PHILLIPS et al. (2000). Ruled pattern: modern glaciers; black: maximal extent during Holocene; shaded: mid-Holocene readvance and/or stillstand; dashed line: maximal extent of glaciers during Oxygen Isotope Stage 3 (OIS-3). völlig eisfreies Indus-Tal argumentiert. Auch HASERODT (1989b) sah das mittlere Indus- sowie das untere Gilgit-Tal, ja sogar das untere Hunza-Tal bis oberhalb von Chalt im Letztglazial als ehemals völlig bzw. streckenweise eisfrei an (Abb. 2). Letztglaziale Seitentalgletscher haben ihm zufolge nur in den Fällen des Astor-Tals und des Rakhiot-Tals am Nanga Parbat und der Täler bei Shatial (850 m ü.M.) bis hinab in das Indus-Tal gereicht und Endmoränen hinterlassen.

Die Vorstellung einer letztglazialen Vergletscherung des mittleren Indus scheint mittlerweile jedoch eher gesichert - lediglich über die Lage des maximalen Eisrandes besteht noch Uneinigkeit (Derbyshire et al. 1984; Desio \& Orombrelli 1971; KuHLE 1996a u. b; NorIN 1925; OWen 1989; SHroder et al. 1989, 1993). Nach den jüngsten Geländebefunden und OSL-Datierungen nach RicharDs et al. (2000) hat der IndusGletscher - vom Hunza- über das Gilgit-Tal in das Indus-Tal hineinreichend - dabei wahrscheinlich bei Shatial geendet (s. Kap. 4.1). Die kosmogenen Datierungen von PHILLIPs et al. (2000) stehen mit dieser Vorstellung einer ausgedehnten Vereisung während des OIS-2 allerdings in Konflikt; die Autoren beschrieben vielmehr zwei Hauptvergletscherungen im OIS-3.

KuHLe (1989, 1996a u. b) rekonstruierte ein ausgedehntes Eisstromnetz und kalkulierte im Bereich der Rakhiot-Brücke eine Eismächtigkeit von 1800-1900 m. Die ausgiebigste Eisausweitung postulierte jedoch $\mathrm{XU}$ DAOMING (1991), der einen vorletztglazialen Eisschild mit dem Karakorum als Zentrum und eine letztglaziale Eiskappe in Höhen oberhalb von $4600 \mathrm{~m}$ ü.M. mit mächtigen Talgletschern für möglich hielt, obschon seine errechnete Schneegrenzdepression nicht gröBer als die anderer Autoren ist (Tab. 3).

\section{Fazit}

Der Vergleich der Verhältnisse im östlichen Hindukusch, im Karakorum und im West-Himalya Pakistans hält folgende Hauptergebnisse fest: 
(1) Mit dem Einsatz der neusten Datierungsmethoden und deren ständiger Präzisierung können zunehmend exaktere Alterseingrenzungen vorgenommen werden. Im allgemeinen scheint sich dabei abzuzeichnen, dass viele vorliegende Chronologien der glazialen Geschichte der Region sehr viel jünger einzustufen sind als bisher angenommen. SHroder (pers. Mitteilung) favorisiert mittlerweile das Modell dreier Hauptvergletscherungen zwischen $\sim 60-50 \mathrm{ky}$, $\sim 40-30 \mathrm{ky}$ und $\sim 9-5 \mathrm{ky}$. Im Lichte der extremen Hebung und der höchsten heute bekannten Erosionsraten der Erde überhaupt erscheinen ihm die oben beschriebenen ausgedehnten Vergletscherungen des Letztglazials allerdings plausibel.

(2) Die glaziale Geschichte mit ihren verschiedenen Phasen und Ereignissen verlief in den drei nordpakistanischen Hochgebirgen Hindukusch, Karakorum und West-Himalaya während des frühen und mittleren Letztglazials synchron. Darüber hinaus scheint das Letztglazial in diesem Raum jedoch asynchron zu dem in der restlichen Nordhemisphäre und dem restlichen Hochasien, wie z. B. dem östlichen Himalaya, verlaufen zu sein. Insbesondere muß das ,regionale" Last Glacial Maximum (rLGM) in das frühe bzw. mittlere letzte Glazial zwischen 70 und 30 ky gestellt werden. Während der ,globalen" Maximalausdehnung (gLGM) waren die nordpakistanischen Hochgebirge aufgrund von Aridität lediglich minimal vergletschert. Erst im Spätglazial/Frühholozän erfolgte ein erneuter Gletschervorstoß, doch war dieser weitaus unbedeutender, denn es handelte sich vornehmlich um Seitentalvergletscherungen. Insgesamt scheint das Holozän relativ synchron zum Geschehen in der übrigen nördlichen Hemisphäre verlaufen zu sein.

Als Ursachen der diagnostizierten Vergletscherungsphasen müssen veränderte Klimabedingungen und hierbei insbesondere veränderte hygrische Verhältnisse angesehen werden. Selbstverständlich üben auch tektonische Bewegungen durch die Erhöhung bzw. Erniedrigung der Reliefenergie auf lokale und vielleicht auch regionale klimatische Verhältnisse Einfluß aus, doch reichen sie als Erklärungsmodell allein nicht aus. Vielmehr scheint es auch zu Verlagerungen des sensiblen asiatischen Monsun-Systems gekommen zu sein, was die asynchronen glazialen Verläufe im westlichen Himalaya-Karakorum-Hindukusch-System auf der einen
Seite und dem östlichen Himalaya und Tibet auf der anderen Seite erklären kann (BENN \& OwEN 1998; Clemens et al. 1991; Emeis et al 1995; RiCHARDS et al. 2000). Schon An Zhisheng et al. (1991) leiteten Schwankungen des ostasiatischen Monsunklimas aus Profilen des chinesischen Lößplateaus ab.

\section{Ausblick}

Es bestehen in allen Untersuchungen nach wie vor Unsicherheiten bzgl. der Ausmaße und der Zeiträume der einzelnen Glaziale bzw. Stadiale in dem Gesamtraum. Es bleibt zu überprüfen, inwieweit den in jüngerer Zeit immer häufiger durchgeführten absoluten Datierungen Vertrauen geschenkt werden kann. Dies gilt um so mehr, wenn zuvor mittels relativer Korrelationen aufgestellte Stratigraphien aufgrund einzelner absoluter Datierungen eine neue Einordnung erfahren sollen. Die Methoden der absoluten Altersdatierung stellen jedoch ein wichtiges Instrument in der Landschafts- und Klimarekonstruktion dar, das nur durch seinen Einsatz perfektioniert werden kann. Weitere Datierungen, vor allem an unsicheren Lokalitäten, sollten daher folgen. Auch andere Methoden wie die Satellitenbildinterpretation mit multitemporalem Ansatz zur Schnee- und Eisbedeckung sowie der Einsatz von digitalen Höhenmodellen können sowohl das rezente glaziale System als auch das Niederschlagssystem besser zu verstehen helfen (BISHOP et al. 1995, 1998; BISHOP \& Shroder 1999). Die Ergebnisse solcher Untersuchungen sollten Eingang finden in Modellrechnungen zur glazialen Vergangenheit und zum Klimageschehen, so wie sie z. B. von BöHNER (1996) durchgeführt wurden oder zur Zeit im Rahmen des International Geological Correlation Programme (IGCP) 415 angestellt werden.

\section{Schriftenverzeichnis}

An Zhisheng, Kulka, G. J., Porter, S. \& Xiao, J. (1991): Magnetic susceptibility evidence of monsoon variations on the loess plateau of Central China during the last 130.000 years. - Quat. Res., 36: 29-36.

Batura Glacier Investigation Group (1976): Investigation report on the Batura Glacier in the Karakoram Mountains, the Islamic Republic of Pakistan (1974-1975). - Batura Investigation Group, Engeneering Headquarters, Beijing.

Batura Glacier Investigation Group (1979): The Batura Glacier in the Karakoram Mountains and its variations. - Sci. Sinica, 22: 959-974.

Benn, D. \& Lehmkuhl, F. (2000): Mass balance and equilibrium-line altitudes of glaciers in high-mountain environments. - Quat. Int., 65/66: 15-29. 
BENN, D. \& OwEN, L. A. (1998): The role of the Indian summer monsoon and the mid-latitude westerlies in Himalayan glaciation: Review and speculative discussion. - J. Brit. Geol. Soc., 155: 353-363.

Bishop, M. P. \& SHroder, J. F. (1999): SPOT panchromatic imagery and neural networks for information extraction in a complex mountain environment. - Geocarto Int., 14: 17-26.

Bishop, M. P., Shroder, J. F., Hickman, B. L. \& CopLAND, L. (1998): Scale-dependent analysis of satellite imagery for characterization of glacier surfaces in the Karakoram Himalaya. - Geomorphology, 21: 217-232.

Bishop, M. P., Shroder, J. F. \& WARD, J. L. (1995): SPOT multispectral analysis for producing supraglacial debris-load estimates for Batura Glacier, Pakistan. - Geocarto Int., 10: 81-90.

BÖHNER, J. (1996): Säkulare Klimaschwankungen und rezente Klimatrends Zentral- und Hochasiens. Göttinger Geogr. Abh., 101; Göttingen.

Brookfield, M. E. (1993): Miocene to Holocene uplift and sedimentation in the northern Himalaya and adjacent areas. - In: SHroder, J. F. [Ed.]: Himalaya to the Sea: Geology, Geomorphology and the Quaternary: 43-71, London.

Buchroithner, M. F. (1985): Geological map of the Tirich Mir (Hindu Kush - Pakistan), scale 1:50.000. - Jb. Geol., B-A.

Burbank, D. W. \& Cheng, K. J. (1991): Relative dating of Quaternary moraines, Rongbuk Valley, Mount Everest, Tibet: Implications for an ice sheet on the Tibetean Plateau. - Quat. Res., 19: 1-18.

Burbank, D. W. \& Johnson, G. D. (1982): Intermontane basin development in the past 4 myr in the north-west Himalaya. - Nature, 298: 432-436.

Clemens, S., Prell, W., Murray, D., Shimmeld, G. \& WeEDon, G. (1991): Forcing mechanisms of the Indian Ocean monsoon. - Nature, 353: 720-725.

Dainelui, G. (1922): Studi sul glaciale. Spedizione Italiane de Filippi nell' Himalaia, Caracorum e Turchestan Cinese (1913-1914). - Seriae II, 5.3.

Derbyshire, E. (1984): Sedimentological analysis of glacial and proglacial debris: a framework for the study of Karakoram glaciers. - In: Miller, K. J. [Ed.]: The International Karakoram Project, 1: 347364; Cambridge.

Derbyshire, E., Li Jijun, Perrot, F. A., Xu Shuying \& WATERS, R. S. (1984): Quaternary glacial history of the Hunza Valley, Karakoram Mountains, Pakistan. - In: Miller, K. J. [Ed.]: The International Karakoram Project, 2: 456-495; Cambridge.

Derbyshire, E. \& Owen, L. A. (1990): Quaternary alluvial fans in the Karakoram Mountains. - In: RACHOCKI, A. H. \& Church, M. [Ed.]: Alluvial fans: A field approach: 27-53; Chichester.

Desio, A. (1975): Notes on the Pleistocene of central Badakhshan. - Geology of Central Badakhshan, Italian Expedition to the Karakorum and Hindu Kush, 3: 339-409; Leiden.

Desio, A. \& Orombelli, G. (1971): Preliminary note on the presence of a large valley glacier in the middle Indus Valley (Pakistan) during the Pleistocene.
- Atti Accad. Nazionale Lincei, 51: 387-392.

Emeis, K.-C., Anderson, D. M., Doose, H., Kroon, D. \& Schulzbull, D. (1995): Sea-surface temperatures and the history of monsoon upwelling in the northwest Arabian Sea during the last 500.000 years. Quat. Res., 43: 355-361.

Finsterwalder, R. (1936): Die Formen der Nanga-Parbat-Gruppe. - Z. Ges. Erdk. Berlin; 71: 321-341.

FinsteriWALDER, R. (1937): Die Gletscher des Nanga Parbat. Glaziologische Arbeiten der Deutschen Himalaya-Expedition 1934 und ihre Ergebnisse. Z. Gletscherk., 25: 57-108.

GARDNER, J. S. \& JONES, N. K. (1993): Sediment transport and yield at the Raikot Glacier, Nanga Parbat, Punjab Himalaya. - In: SHRoder, J. F. [Hrsg.]: Himalaya to the sea: Geology, Geomorphology and the Quaternary: 184-197; London.

Goudie, A. S., Jones, D. K. C. \& Brunsden, D. (1984): Recent fluctuations in some glaciers of the western Karakoram mountains, Pakistan. - In: Miller, K. J. [Hrsg.]: The International Karakoram Project, 2: 411-455; Cambridge.

GrötzBach, E. \& RATHJENS, C. (1969): Die heutige und die jungpleistozäne Vergletscherung des afghanischen Hindukusch. - Z. Geomorph. N.F., Suppl.Bd., 8: 58-75.

HASERODT, K. (1968): Zur quartären Vergletscherung des pakistanischen Hindukusch (Chitral). - Eiszeitalter u. Gegenwart, 19: 302-303.

HASERODT, K. (1982): Die quartäre Vergletscherung am pakistanischen Hindukusch (Chitral). - Sitzungsber. Mitt. Braunschweiger Wiss. Ges., Sonderbd., 6: 2527 (und Karte).

Haserodt, K. (1984): Aspects of the actual climatic conditions and historic fluctuations of glaciers in Western Karakoram. - J. Central Asia, 7: 77-94.

Haserodt, K. (1989a): Chitral (pakistanischer Hindukusch). Strukturen, Wandel und Probleme eines Lebensraumes im Hochgebirge zwischen Gletschern und Wüste. - In: HasERODT, K. [Hrsg.]: Hochgebirgsräume Nordpakistans im Hindukusch, Karakorum und Westhimalaya, Beitr. Mat. Reg. Geogr., 2: 43-180; Berlin.

HASERODT, K. (1989b): Zur pleistozänen und postglazialen Vergletscherung zwischen Hindukusch, Karakorum und Westhimalaya. - In: HAserodt, K. [Hrsg.]: Hochgebirgstäume Nordpakistans im Hindukusch, Karakorum und Westhimalaya, Beitr. Mat. Reg. Geogr., 2: 181-233; Berlin.

Heuberger, H. (1980): Die Schneegrenze als Leithorizont in der Geomorphologie. - In: JeNTSCH, C. \& LiedTKE, H. [Hrsg.]: Höhengrenzen in Hochgebirgen, Arb. Geogr. Inst. Univ. Saarlandes, 29: 35-48; Saarbrücken.

Hewit, K. (1961): Karakoram glaciers and the Indus. Indus, 2: 4-14.

Holmes, J. A. (1993): Present and past patterns of glaciation on the northwest Himalaya: Climatic, tectonic and topographic controls. - In: SHRODER, J. F. [Hrsg.]: Himalaya to the Sea: Geology, Geomorphology and the Quaternary: 72-90; London. 
Holmes, J. A. \& Street-Perrott, F. A. (1989): The Quaternary glacial history of Kashmir, north-west Himalava: A revision of de Terra and Paterson's Sequence. - Z. Geomorph. N.F., Suppl.-Bd., 76: 195-212.

JANOTTA, A. (1991): Thermolumineszenzdatierungen als chronometrischer Beitrag zur stratigraphischen Beschreibung von Lößprofilen. - Düsseldorfer Geogr. Schr., 30; Düsseldorf.

JanotTA, A. \& BrunotTe, E. (1995): Thermoluminescence dating - methodical deficits and insufficient landform analysis. - Z. Geomorph. N. F., Suppl.Bd., 99: 29-39.

KAMP, U. (1999): Jungquartäre Geomorphologie und Vergletscherung im östlichen Hindukusch, Chitral, Nordpakistan. - Berliner Geogr. Stud., 50; Berlin.

KAMP, U. (2001a): Die jungquartäre Vergletscherung Chitrals im östlichen Hindukusch, Pakistan. - Z. Gletscherk. Glazialgeol., 36: 81-106.

KAmp, U. (2001b): Jungquartäre Terrassen und Talentwicklung in Chitral, östlicher Hindukusch. - Z. Geomorph. N.F., 45, (im Druck).

Kerschner, H. (1990): Methoden der Schneegrenzbestimmung. - In: LiedtKE, H. [Hrsg.]: Eiszeitforschung: 299-311; Darmstadt.

KICK, W. (1985): Geomorphologie und rezente Gletscheränderungen in Hochasien. - In: HARTL, W. \& Engelschalk, W. [Hrsg.]: Geographie, Naturwissenschaft und Geisteswissenschaft, Regensburger Geogr. Schr., 19/20: 53-77; Regensburg.

KICK, W. (1996): Forschung am Nanga Parbat. Geschichte und Ergebnisse. - In: KICK, W. [Hrsg.]: Forschung am Nanga Parbat, Geschichte und Ergebnisse, Beitr. Mat. Reg. Geogr., 8: 1-134; Berlin.

Kunle, M. (1986): The upper limit of glaciation in the Himalayas. - Geo J., 13: 331-346.

Kunle, M. (1989): Die Inlandvereisung Tibets als Basis einer in der Globalstrahlungsgeometrie fußenden, reliefspezifischen Eiszeittheorie. - Petermanns Geogr. Mitt., 133: 265-285.

Kuhle, M. (1991): Observations supporting the Pleistocene inland glaciation of High Asia. - Geo J., 25: 133-231.

Kunle, M. (1993): Eine Autozyklentheorie zur Entstehung und Abfolge der quartären Kalt- und Warmzeiten auf der Grundlage epirogener und glazialisostatischer Bewegungsinterferenzen im Bereich des tibetanischen Hochlandes. - Petermanns Geogr. Mitt., 137: 133-152.

Kuhle, M. (1996a): New findings concerning the ice age (Last Glacial Maximum) glacier cover of the East-Pamir, of the Nanga Parbat up to the Central Himalaya and of Tibet, as well as the age of the Tibetan inland ice. - Geo J., 42: 87-257.

KuHLE, M. (1996b): Rekonstrukton der maximalen eiszeitlichen Gletscherbedeckung im Nanga-ParbatMassiv $\left(35^{\circ} 05^{\prime}-40^{\prime} \mathrm{N} / 74^{\circ} 20^{\prime}-75^{\circ} \mathrm{E}\right)$. - In: KICK, W. [Hrsg.]: Forschung am Nanga Parbat, Beitr. Mat. Reg. Geogr., 8: 135-156; Berlin.

Kunle, M. (1998): Reconstruction of the 2.4 million $\mathrm{km}^{2}$ Late Pleistocene ice sheet on the Tibetean plateau and its impact on the global climate. - Quat. Int., 45/46: 71-108.
Loewe, F. (1924): Die Eiszeit in Kaschmir, Baltistan und Ladakh. - Z. Gesell. Erdk. Berlin: 42-53.

Louis, F. (1955): Schneegrenze und Schneegrenzbestimmung. - Geogr. Taschenbuch 1954/1955:414-418; Wiesbaden

MeINERS, S. (1995): Exemplary observations concerning historical to neoglacial glacier stades in the catchment area of the NW-Karakoram (Hispar Muztagh, Batura Muztagh, Rakaposhi Range) and the boundary of ecumene. - Abstracts for the International Symposium on "Karakorum-Hindukush-Himalaya: Dynamics of change", 29.9.-2.10.1995, Islamabad, Pakistan: 28; Islamabad.

Meiners, S. (1997): Historical to Post Glacial glaciation and their differentiation from the Late Glacial period on examples of the Tian Shan and the N.W. Karakorum. - Geo J., 42: 259-302.

Mercer, J. H. (1975): Glaciers of the Karakoram. - In: FIELD, W. W. [Hrsg.]: mountain Glaciers of the Northern Hemisphere, 103 S.; Hannover, New Hampshire.

Miller, K. J. [Hrsg.] (1984): The International Karakoram Project (Volume 1 and 2). - Cambridge.

Mirwald, P. \& Roemer, H. (1967): Beobachtungen im Wakhan (NE-Afghanistan). - Erdk., 21: 48-57.

Norin, E. (1925): Preliminary notes on the Late Quaternary glaciation of the North Western Himalaya. - Geogr. Annaler, 7: 165-194.

Oestreich, K. (1906): Die Täler des nordwestlichen Himalaya. - Petermanns Geogr. Mitt., 155: 12-42.

Owen, L. A. (1988): Terraces, uplift and climate, Karakoram mountains, Northern Pakistan. - Unveröffentlichte PhD-Thesis, University of Leicester.

Owen, L. A. (1989): Terraces, uplift and climate in the Karakoram Mountains, northern Pakistan: Karakoram intermontane basin evolution. - Z. Geomorph. N.F., Suppl.-Bd., 76: 117-146.

Owen, L. A. \& Derbyshire, E. (1989): The Karakoram glacial depositional system. - Z. Geomorph. N.F., Suppl.-Bd., 76: 33-73.

Owen, L. A., Derbyshire, E. \& Fort, M. (1998): The Quaternary glacial history of the Himalaya. - Quat. Proc., 6: 91-120.

Owen, L. A., Scott, C. H. \& Derbyshire, E. (2000): The Quaternary glacial history of Nanga Parbat. Quat. Int., 65/66: 63-79.

Owen, L. A., White, B. J., Rendell, H. \& Derbyshire, E. (1992): Loessic silt deposits in the western Himalayas: Their sedimentology, genesis and age. - Catena, 19: 493-509.

Owen, L., Kamp, U., Spencer, J. \& Haserodt, K. (2002): Timing and style of Late Quaternary glaciation in the eastern Hindu Kush, Chitral, northern Pakistan: a review and revision of the glacial chronology based on new optically stimulated luminescence dating. - Quat. Int., (in Druck).

Paffen, K.-H., Pillewitzer, W. \& Schneider, H.-J. (1956): Forschungen im Hunza-Karakorum. Erdk., 10: 1-33.

Phillips, W. M., Sloan, V. M., Shroder, J. F., Sharma, P., Clarke, M. L. \& Rendell, H. M. (2000): Asynchronous glaciation at Nanga Parbat, northwest- 
ern Himalaya Mountains, Pakistan. - Geology, 28: 431-434.

PilLEWIZER, W. (1962): Vergletscherung und Schneegrenze in Hochasien. - Petermanns Geogr. Mitt., 106: 186-187.

PorTer, S. C. (1970): Quaternary glacial record in Swat Kohistan, West Pakistan. - Bull. Geol. Soc. America, 81: 1421-1446.

Richards, B. W., OwEN, L. A. \& RHODEs, E. J. (2000): Timing of Late Quaternary glaciations in the Himalayas of northern Pakistan. - J. Quat. Sci., 15: 283297.

RÖTHLISBERGER, F. (1986): 10.000 Jahre Gletschergeschichte der Erde, Teil 1 und 2. - Frankfurt a. M.

Röthlisberger, F. \& GeYh, M. A. (1985): Glacier variations in Himalayas and Karakorum. - Z. Gletscherk. Glazialgeol., 21: 237-249.

SCHNEIDER, H. J. (1959): Zur diluvialen Geschichte des NW-Karakorum. - Mitt. Geogr. Ges. München, 44: 201-216.

SCHNEIDER, H. J. (1969): Minapin - Gletscher und Menschen im NW-Karakorum (Erläuterungen zur Expeditionskarte 1 : 50.000). - Die Erde, 100: 266286.

Sсотт, C. H. (1992): Contemporary sediment transfer in the Himalayan glacial system: Implications for the interpretation of the Quaternary record. - Unveröffentlichte $\mathrm{PhD}$-Thesis, University of Leicester.

Searle, M. P. (1991): Geology and tectonics of the Karakoram mountains. - Chichester.

Searle, M. P. \& Khan, M. A. [Hrsg.] (o. A.): Geological map of northern Pakistan and adjacent areas of northern Ladakh and western Tibet (Western Himalaya, Salt Ranges, Kohistan, Karakoram, Hindu Kush) $1: 650000$.

Shakleton, N. J. \& Obdyke, N. D. (1973): Oxygene isotope and palaeomagnetic stratigraphy of equatorial Pacific core V28-238. - Quat. Res., 3: 39-55.

ShI YAFENG \& Zhang Xiangsong (1984): Some studies of the Batura Glacier in the Karakoram mountains. - In: Miller, K. J. [Hrsg.]: The International Karakoram Project, 1: 51-63; Cambridge.

Shi Yafeng, Zheng, B. \& Li, S. (1992): Last glaciation and maximum glaciation in the Qinghai-Xizang (Tibet) Plateau: A controversy to M. Kuhle's ice sheet hypothesis. - Z. Geomorph. N.F., Suppl.-Bd., 84: 19-35.

Shroder, J. F. (1984): Batura glacier terminus, 1984, Karakorum Himalaya. - Geol. Bull. Univ. Peshawar, 17: 119-126.

SHroder, J. F. (1993): Himalaya to the sea: Geomorphology and the Quaternary of Pakistan in the regional context. - In: SHRoder, J. F. [Hrsg.]: Himalaya to the sea: Geology, Geomorphology and the Quaternary: 1-42; London.

Shroder, J. F., Bishop, M. P., Copland, L. \& Sloan, V. F. (2000): Debris-covered glaciers and rock glaciers in the Nanga Parbat Himalaya, Pakistan. - Geogr. Annaler, 82A: 1-10.

Shroder, J. F., Khan, M. S., Lawrence, R. D., Madin, I. P. \& Higgins, S. M. (1989): Quaternary glacial chronology and neotectonics in the Himalaya of north- ern Pakistan. - In: Malinconico, C. [Hrsg.]: Geology of the Western Himalayas, Geol. Soc. America Spec. Pap., 232: 275-294; Boulder.

Shroder, J. F., Owen, L. A. \& Derbyshire, E. (1993): Quaternary glaciation of the Karakoram and Nanga Parbat Himalaya. - In: Shroder, J. F. [Hrsg.]: Himalaya to the Sea: Geology, Geomorphology and the Quaternary: 132-158; London.

Slodn, V., Phillips, W. M., Shroder, J. F. \& Sharma, P. (1998): Asynchronous maximum advances of mountain glaciers in the Pakistan Himalaya. - GSA Abstr. Progr., 30: A-229.

Terra, H. DE (1938): Der eiszeitliche Zyklus in Südasien und seine Bedeutung für die menschliche Vorgeschichte. - Z. Ges. Erdk. Berlin: 285-296.

TrinkLeR, E. (1932): Geographische Forschungen im westlichen Zentralasien und Karakorum-Himalaya. - Wissenschaftliche Ergebnisse der Dr. Trinklerschen Zentralasien-Expedition: 1; Berlin.

Troll, C. (1938): Reliefenergie und Vergletscherung in der Nanga Parbat-Gruppe. - Z. Gletscherk., 26: 303307.

Visser, P. C. (1928): Von den Gletschern am obersten Indus. - Z. Gletscherk., 16: 169-229.

WADIA, D. N. (1932/33): Note on the geology of Nanga Parbat (Mt. Diamir), and adjoining portions of Chilas, Gligit District, Kashmir. - Rec. Geol. Survey India, 66: 212-234.

Wiche, K. (1958): Die österreichische Karakorum-Expedition 1958. - Mitt. Geogr. Ges. Wien, 10: 280294.

WICHE, K. (1959): Klimamorphologische Untersuchungen im westlichen Karakorum. - Verh. Dt. Geographentages, 32: 190-203.

WissmanN, H. VON (1959): Die heutige Vergletscherung und Schneegrenze in Hochasien mit Hinweisen auf die Vergletscherung der letzten Eiszeit. - Akad. Wiss. Lit. Mainz, Abh. Math.-Nat. Kl., 14: 11031407.

Xu DaOMING (1991): Quaternary glaciation of the north slope of Karakorum mountains. - Geo J., 25: 233242.

Zeitler, P. K., Johnson, N. M., Naeser, C. W. \& TAHIRKHELI, R. A. K. (1982): Fission-track evidence for Quaternary uplift of the Nanga Parbat region, Pakistan. - Nature, 298: 255-257.

Zeitler, P. K., Johnson, N. M., Tahirkheli, R. A. K. \& NAESER, C. W. (1981): Nanga Parbat massif rates and uplift. - GSA Abstr. Progr., 13: 587.

Zhang Xiangsong (1984): Recent variations of some glaciers in the Karakoram mountains. - In: MiLLER, K. J. [Hrsg.]: The International Karakoram Project, 1: 39-50; Cambridge.

Zhang Xiangsong \& Shi Yafeng (1980): Changes of the Batura Glacier in the Quaternary and recent times. - Professional Papers on the Batura Glacier, Karakoram Mountains: 173-190; Beijing.

ZHENG BENXING (1989): Controversy regarding the existence of a large ice sheet on the Qinghai-Xizang (Tibetean) Plateau during the Quaternary period. Quat. Res., 32: 121-123. 